
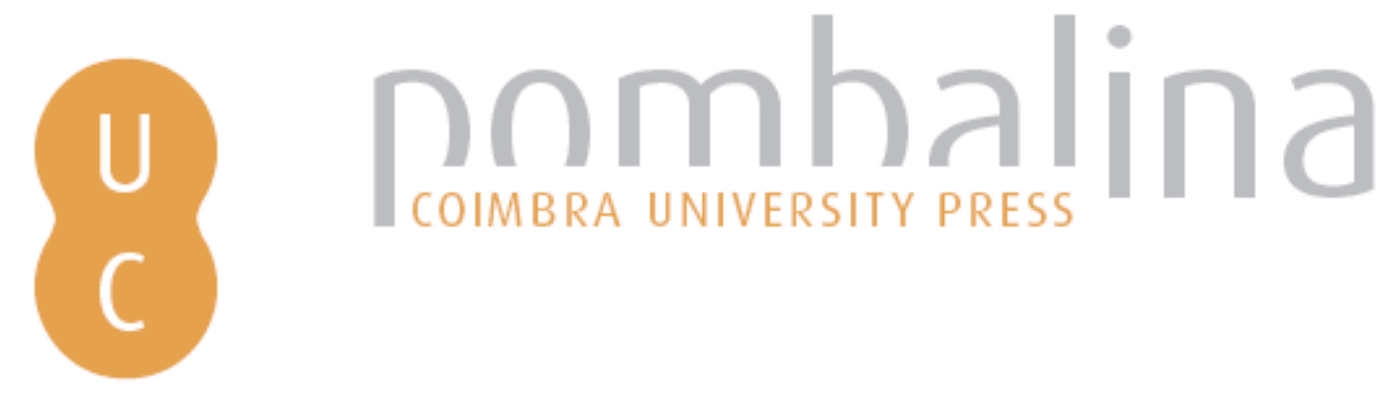

\title{
Hidrogeles de ciclodextrinas para administración de fármacos.
}

Autor(es): $\quad$ Santos, José F. Rosa Dos; Torres Labandeira, Juan J.; Alvarez Lorenzo, Carmen; Concheiro, Angel

Publicado por: Imprensa da Universidade de Coimbra

URL

persistente: URI:http://hdl.handle.net/10316.2/36864

DOI: $\quad$ DOI:http://dx.doi.org/10.14195/978-989-26-0881-5_1

Accessed : $\quad$ 26-Apr-2023 11:24:42

A navegação consulta e descarregamento dos títulos inseridos nas Bibliotecas Digitais UC Digitalis, UC Pombalina e UC Impactum, pressupõem a aceitação plena e sem reservas dos Termos e Condições de Uso destas Bibliotecas Digitais, disponíveis em https://digitalis.uc.pt/pt-pt/termos.

Conforme exposto nos referidos Termos e Condições de Uso, o descarregamento de títulos de acesso restrito requer uma licença válida de autorização devendo o utilizador aceder ao(s) documento(s) a partir de um endereço de IP da instituição detentora da supramencionada licença.

Ao utilizador é apenas permitido o descarregamento para uso pessoal, pelo que o emprego do(s) título(s) descarregado(s) para outro fim, designadamente comercial, carece de autorização do respetivo autor ou editor da obra.

Na medida em que todas as obras da UC Digitalis se encontram protegidas pelo Código do Direito de Autor e Direitos Conexos e demais legislação aplicável, toda a cópia, parcial ou total, deste documento, nos casos em que é legalmente admitida, deverá conter ou fazer-se acompanhar por este aviso.

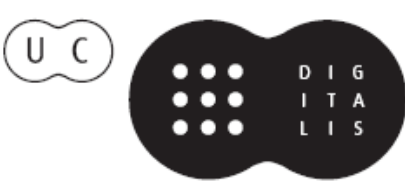


IOMATERIAIS APLICADOS AO DESENVOLVIMENTO DE SISTEMAS TERAPÊUTICOS AVANÇADOS

IOMATERIALES APLICADOS AL DISEÑO DE SISTEMAS TERAPÉUTICOS AVANZADOS

Hermínio C. de Sousa Mara E. M. Braga Alejandro Sosnik (editores) 


\section{A P ÍTULO 1 .}

\section{H I D ROG ELES DE CICLODEXTRINAS}

\section{PARA ADMINISTRACIÓn DE FÁRMACOS}

José F. Rosa Dos Santos, Juan J. Torres Labandeira, Carmen Alvarez Lorenzo, Angel Concheiro Departamento de Farmacia y Tecnología Farmacéutica, Facultad de Farmacia, Universidad de Santiago de Compostela, 15782-Santiago de Compostela (España)

\section{Resumen:}

Las ciclodextrinas (CDs) son oligosacáridos cíclicos constituidos por anillos de seis a ocho unidades de glucosa ligadas por enlaces $\alpha(1-4)$, de manera que forman un toroide en el que los grupos hidrofílicos quedan expuestos hacia el exterior. Esta conformación determina que puedan formar complejos de inclusión con fármacos de naturaleza diversa, que presenten en su estructura grupos poco polares. Tradicionalmente, las CDs se han utilizado dispersas de manera individualizada para mejorar las propiedades fisicoquímicas y biofarmacéuticas de gran número de fármacos. Esta aproximación tiene la limitación de que la dilución en medio fisiológico suele desencadenar una rápida decomplejación, con el consiguiente riesgo de precipitación. La incorporación de CDs en entramados poliméricos conduce a sinergias que incrementan considerablemente sus prestaciones. Las CDs permiten modular las propiedades del entramado y dotarlo de un nuevo mecanismo de incorporación del fármaco y de control del proceso de cesión; por su parte, el confinamiento de las CDs en un entramado que, cuando se administra al organismo, se disgrega lentamente hace que se mantenga una elevada 
concentración local de CDs que ralentiza la decomplejación del fármaco. En este capítulo se revisan las posibilidades que ofrece la incorporación en entramados poliméricos de CDs libres, la formación de poli(pseudo) rotaxanos con CDs químicamente unidas, y la incorporación de CDs al esqueleto del entramado polimérico o como estructuras colgantes, en los campos de la tecnología farmacéutica y la medicina regenerativa.

Palabras clave: ciclodextrina; polipseudorotaxano; gel físicamente reticulado; hidrogel químicamente reticulado; monómero de ciclodextrina; cesión controlada.

\begin{abstract}
:
Cyclodextrins (CDs) are cyclic oligosaccharides consisting of six to eight ring glucose units linked by $\alpha(1-4)$ bonds, so as to form a toroid in which the hydrophilic groups are exposed to the outside. This arrangement determines that CDs can form inclusion complexes with a variety of drugs bearing low polar groups. Traditionally, CDs have been used individually dispersed to improve the physicochemical and biopharmaceutical properties of many drugs. This approach has the limitation that the dilution in physiological medium triggers rapid rupture of the complexes, with the risk of drug precipitation. Incorporation of CDs into polymeric networks leads to synergies that greatly increase their performance. CDs enable tuning of network properties and endow it with a new mechanism of drug incorporation and control of release; on the other hand, the confinement of the CDs in a network which, when administered to the body, slowly disintegrates provides a high local concentration of CDs which slows down decomplexation. In this chapter the possibilities of incorporation into polymer networks of free CDs, the formation of poly(pseudo)rotaxanes with chemically bonded CDs, grafting of CDs to the polymer backbone or as pendant structures, are reviewed in the context of pharmaceutical technology and regenerative medicine.
\end{abstract}

Keywords: cyclodextrin; poly(pseudo)rotaxane; physical gel; chemically cross-linked hydrogel; cyclodextrin monomer; controlled release. 


\subsection{Introducción}

Para que un fármaco se absorba y pueda alcanzar su lugar de acción debe presentar un adecuado balance entre su solubilidad en medio acuoso y su capacidad para atravesar las membranas biológicas. La mayoría de los fármacos considerados como esenciales por la Organización Mundial de la Salud, y de las nuevas moléculas candidatas a convertirse en fármacos, presentan un carácter marcadamente lipofílico y, consecuentemente, una baja hidrosolubilidad $[1,2]$. También es frecuente que los fármacos planteen problemas de estabilidad y de toxicidad. Todo ello dificulta el desarrollo de formas de dosificación eficaces y seguras, y obliga a buscar estrategias que permitan alcanzar las concentraciones requeridas en el lugar de acción sin comprometer la seguridad de los tratamientos [3,4]. La posibilidad de incorporar ciclodextrinas (CDs) a una gran variedad de estructuras poliméricas abre interesantes perspectivas para el desarrollo de sistemas hidrofílicos capaces de incorporar, formando complejos de inclusión, fármacos hidrofóbicos o hidrofílicos para cederlos de manera controlada [5-7].

\subsection{Estructura y propiedades de las ciclodextrinas}

Las CDs son oligosacáridos cíclicos constituidos por anillos de seis a doce unidades de glucosa ligadas por enlaces $\alpha(1-4)$ (Figura 1.1). Desde el descubrimiento, hace más de 100 años, de las CDs naturales $\alpha$ ( 6 unidades), $\beta$ ( 7 unidades) y $\gamma$ ( 8 unidades $)$, se han puesto a punto nuevas técnicas de producción y purificación y numerosos métodos de preparación de derivados, lo que ha permitido incrementar sus aplicaciones en el campo farmacéutico [8]. La $\beta$-CD se disuelve en agua con dificultad debido a su tendencia a formar puentes de hidrógeno intramoleculares. La sustitución de algunos grupos hidroxilo por otros grupos, hidrofílicos o hidrofóbicos, conduce a la formación de derivados más hidrosolubles [9,10]. La solubilidad, la capacidad de la CD para formar complejos con otras sustancias y 
otras propiedades de interés práctico dependen de la naturaleza del sustituyente y del grado de sustitución [6,10-12]. La $\alpha-, \beta-$ y $\gamma$-CD y los derivados 2-hidroxipropil- $\beta$-CD (HP- $\beta$-CD), la sulfobutil- $\beta$-CD y la metil- $\beta$-CD (M- $\beta-C D)$ cuentan con la aprobación de las agencias reguladoras para ser utilizadas como excipientes farmacéuticos. El grado de sustitución de las $C D$ de calidad farmacéutica es 4.5-7 para la HP- $\beta$-CD (0.65-1 grupos hidroxipropilo por unidad de glucosa), 7 para la sulfobutil- $\beta-C D$, y 4-12 para la M- $\beta$-CD (0.57-1.8 grupos metilo por unidad de glucosa).
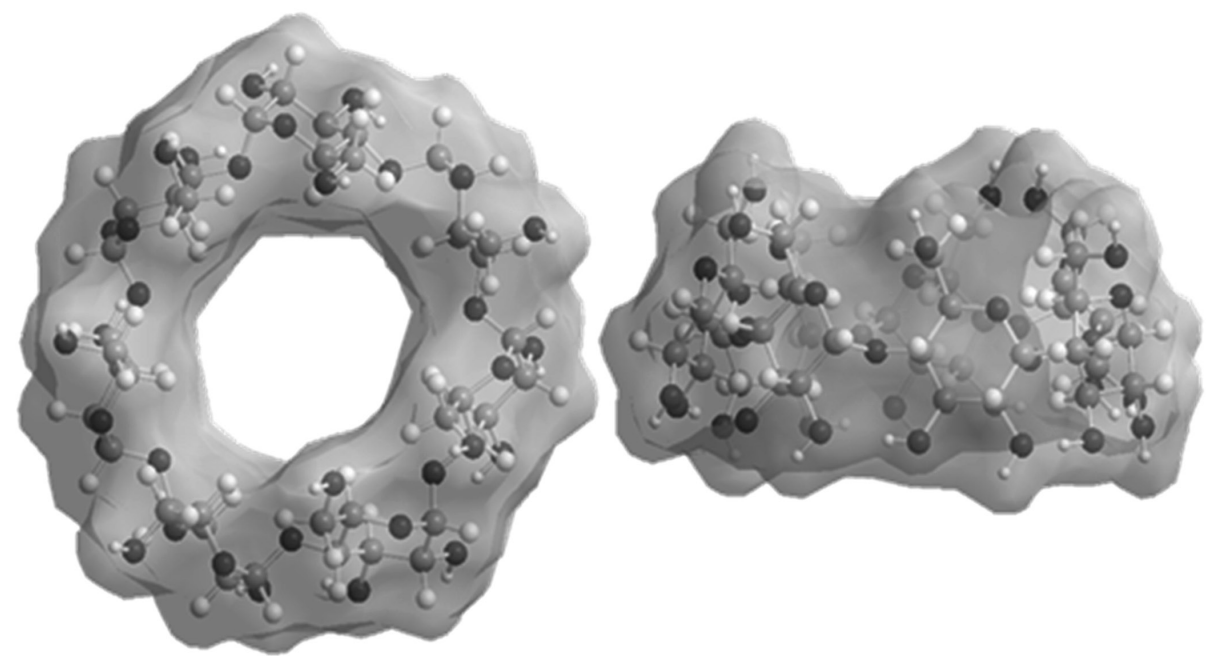

Figura 1.1. Representación espacial esquemática de la $\beta$-ciclodextrina (vista frontal y lateral).

Las CDs tienen forma de cono truncado hueco de $7.9 \AA$ de altura y diámetro máximo comprendido entre 4.7 y $8.3 \AA$ A (Figura 1.1). La cara externa de la cavidad es hidrofílica, mientras que la cara interna es hidrofóbica. Las CDs pueden formar complejos de inclusión con moléculas poco polares capaces de penetrar total o parcialmente en su cavidad, produciéndose la complejación por sustitución de las moléculas de agua inicialmente en el interior de las CDs. En la complejación intervienen interacciones hidrofóbicas, electrostáticas y de van der Waals, así como puentes de hidrogeno y cambios conformacionales, 
estableciéndose un rápido equilibrio entre las moléculas que se encuentran libres en el medio y las que están alojadas en el interior de la cavidad [13]. La adición de cosolventes o polímeros y los cambios de $\mathrm{pH}$ o de temperatura pueden desplazar el equilibrio hacia la complejación o la decomplejación [14-17]. La formación de complejos con CDs puede ser útil para corregir las propiedades organolépticas, incrementar la solubilidad aparente y mejorar la estabilidad de los fármacos $[12,18,19]$. En estado sólido, los complejos suelen presentar estructura amorfa, con lo que la velocidad de disolución del fármaco se incrementa considerablemente.

Por su elevado tamaño e hidrofilia, las CD y los complejos CDfármaco no atraviesan las membranas biológicas [20]. No obstante, los complejos pueden actuar en la proximidad de la membrana como reservorios que proporcionan elevadas concentraciones aparentes de fármaco, lo que facilita el paso del fármaco libre por difusión. Además, los complejos difunden mejor que las moléculas de fármaco libre a través de las capas acuosas asociadas a la superficie de la mucosa. Para que se mantenga el equilibrio de complejación, a medida que el fármaco va atravesando la membrana, se produce una progresiva decomplejación. Además, la complejación puede estabilizar el fármaco evitando su degradación en la zona de absorción. Las CDs pueden actuar como promotores de la absorción, extrayendo componentes lipófilos de la membrana [21] (Figura 1.2). Los complejos también se pueden utilizar para prevenir efectos secundarios locales, como irritación ocular, gastrointestinal o dérmica [22]. 


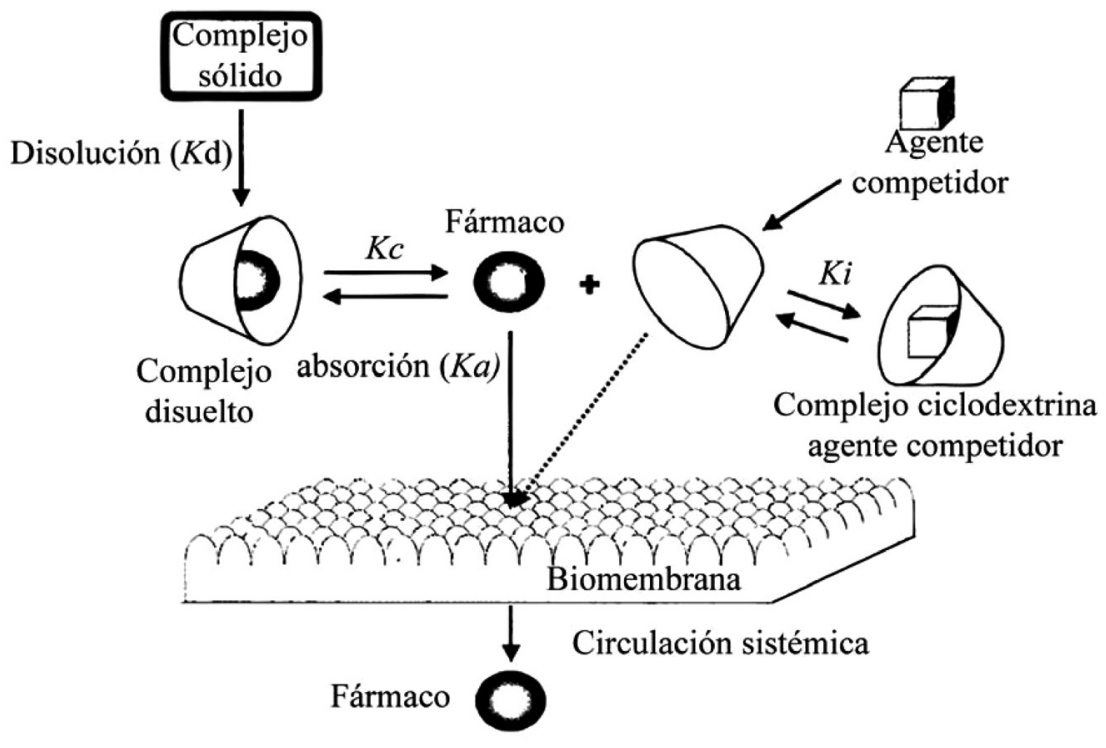

Figura 1.2. Esquema del proceso de disolución y absorción de un fármaco a partir de un complejo de inclusión, y competencia por la complejación de los componentes de la membrana. Adaptado de referencia [23] con permiso de Pharmaceutical Society of Japan.

Esta variedad de funcionalidades y aplicaciones junto con la amplia disponibilidad de datos que prueban la seguridad de las CDs [20] y la progresiva reducción que se está produciendo en los precios de estos excipientes, explican que el número de medicamentos que las incorporan se haya incrementado en los últimos años de una manera muy notable [5,12,23-26]. En la actualidad, están comercializados en el mundo unos cuarenta medicamentos basados en complejos con CDs, para administración oral, sublingual, nasal, ocular, tópica y parenteral [8,12,27]. Además, el interés por las CDs se ha visto potenciado por la adopción por la FDA y la EMA del Sistema de Clasificación Biofarmacéutica (SCB) de fármacos que se administran por vía oral [28]. El SCB distingue cuatro clases de fármacos en función de la solubilidad y la velocidad de disolución de la dosis terapéutica y de la capacidad para atravesar las membranas biológicas [29,30] (Figura 1.3). Las CDs pueden incrementar la solubilidad y la permeabilidad de fármacos de Clase II y de Clase IV, desplazándolos a la Clase I [28]. 


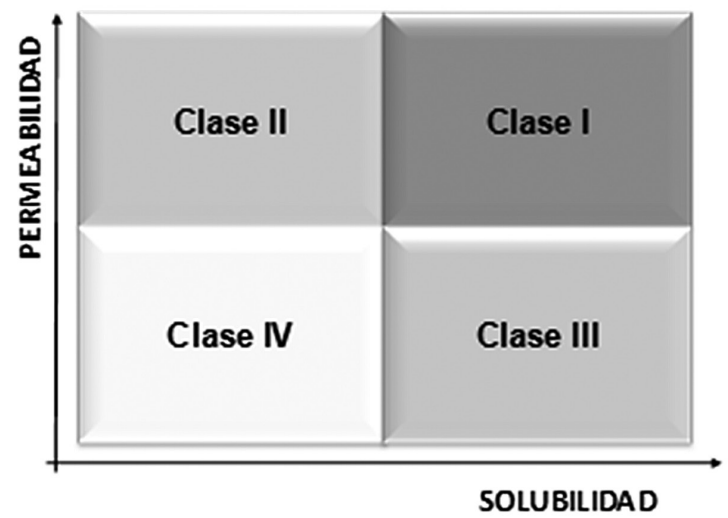

Figura 1.3. Sistema de Clasificación Biofarmacéutica (SCB) de fármacos que se administran por vía oral.

Los fármacos que se encuentran formando complejos de inclusión con CDs experimentan una cesión rápida cuando se administran al organismo, ya sea en forma líquida o sólida, al diluirse los complejos en los fluidos biológicos [31-33]. Esta rápida decomplejación puede resultar ventajosa para desarrollar sistemas de liberación inmediata, facilitando la absorción de glucósidos cardiotónicos, analgésicos o antiepilépticos en situaciones de emergencia [34]. Las CDs con sustituyentes anfifílicos dan lugar a agregados supramoleculares o nanosferas que pueden incorporar fármacos hidrofóbicos en proporciones elevadas. El fármaco también se libera rápidamente hacia el medio acuoso a partir de estas nanoestructuras [35-37]. Por otra parte, las CDs se pueden modificar con grupos ionizables o hidrofóbicos para dotarlas de capacidad para modificar la cesión [6]. Por ejemplo, la solubilidad de CDs modificadas con grupos ácido carboxílico, como la O-(carboximetil)-O-etil- $\beta-\mathrm{CD}$, depende del $\mathrm{pH}$ por lo que resultan útiles para preparar formas entéricas [38]. Los conjugados CD-fármaco tienen también un gran potencial en el desarrollo de formas de liberación colónica [39]. Para conseguir una liberación lenta con fármacos de elevada hidrosolubilidad se puede acudir a CDs con sustituyentes alquílicos o acilados, si bien su capacidad para regular la cesión se ve limitada por la decomplejación relativamente rápida 
que se produce como consecuencia de la dilución de las formulaciones $[40,41]$. Puesto que el equilibrio depende fundamentalmente de la concentración local de $\mathrm{CD}$, una estrategia muy atractiva para controlar la cesión consiste en incorporar el complejo a una estructura que minimice la dilución.

A diferencia de lo que ocurre en los sistemas en los que los complejos CD-fármaco no tienen restringida su movilidad, que ceden el fármaco a una velocidad dependiente del proceso de dilución, la incorporación de CDs a entramados poliméricos o hidrogeles permite controlar la liberación regulando la difusión y/o la afinidad del fármaco por las CDs inmovilizadas [5]. Los hidrogeles son materiales biocompatibles muy versátiles que encierran un gran potencial como componentes de sistemas de liberación de medicamentos para la práctica totalidad de las vías de administración [42]. Los hidrogeles físicamente reticulados controlan la cesión por el efecto que ejerce la viscosidad sobre la difusión, pero su capacidad de regulación no es tan elevada como cabría esperar de los altos valores de macroviscosidad (viscosidad aparente) que presentan, puesto que la variable crítica es la viscosidad del microentorno a través del que debe difundir el fármaco [43,44]. Como consecuencia de ello, es frecuente que no se consiga un control eficaz de la cesión con hidrogeles muy viscosos. La incorporación de CDs hace que el mayor volumen hidrodinámico del complejo ralentice la difusión. Los hidrogeles químicamente reticulados ofrecen mayores posibilidades de control de la cesión. El tamaño de malla del entramado se puede mantener inalterado en el transcurso del proceso o bien modificarse por efecto de estímulos o por reacciones de degradación enzimática [45]. Desde un punto de vista práctico, la utilidad de los hidrogeles se ve limitada por su escasa afinidad hacia los fármacos lipofílicos, que impide que se incorporen en cantidad suficiente, y por el deficiente control de la liberación de los fármacos hidrofílicos. La unión covalente de CDs a hidrogeles químicamente reticulados puede dotar al entramado de afinidad por ciertos fármacos. En la figura 1.4 se esquematizan diferentes formas de incorporación de CDs a entramados poliméricos. 


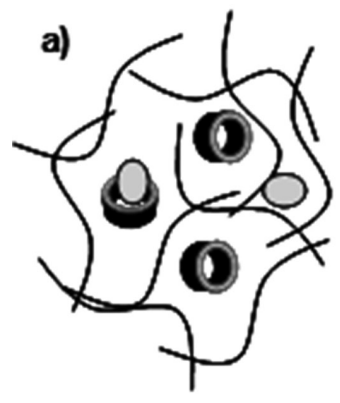

d)

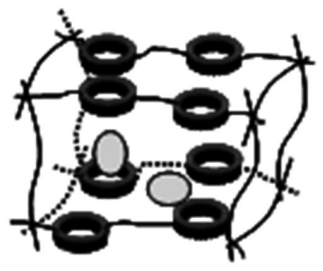

b)

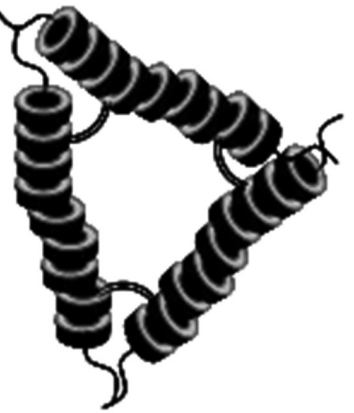

c)

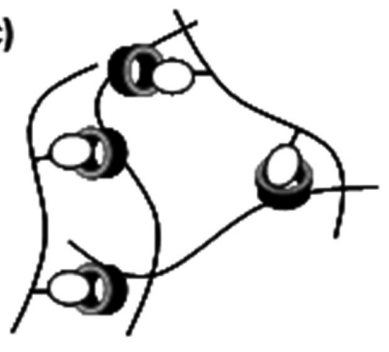

e)

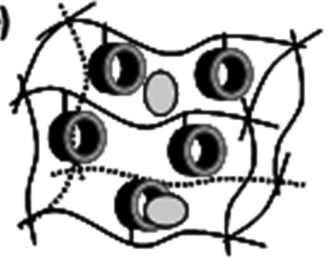

Figura 1.4. Distintas formas en las que se pueden encontrar las CDs formando parte de entramados poliméricos: a) CDs libres; b) poli(pseudo)rotaxanos con las CDs químicamente unidas; c) CDs formando parte de las cadenas poliméricas y actúando como agentes reticulantes que unen varias cadenas poliméricas; d) CDs formando parte del entramado tridimensional del hidrogel; y e) CDs "colgantes" de la estructura del entramado.

\subsection{CDs libres en entramados poliméricos}

La dispersión de CDs en matrices sólidas o semisólidas (Figura 1.4a) es un recurso muy útil para modificar la velocidad de cesión y modular la biodisponibilidad. Si el fármaco se incorpora en cantidades muy altas, las CDs pueden promover la cesión, incrementando la proporción de fármaco disuelto y en condiciones de difundir. Por el contrario, si el fármaco se encuentra en proporciones bajas, de manera que una vez que el sistema se hidrata la concentración sea inferior a su coeficiente de solubilidad, la complejación reduce la concentración de fármaco libre y dificulta la difusión como consecuencia del mayor tamaño de los complejos [46]. Por ejemplo, la incorporación de un 1\% de HP- $\beta$-CD o M- $\beta$-CD a hidrogeles físicos de hidroxipropilmetilcelulosa (HPMC 4000 cPs) facilita la solubilization de melatonina y promueve su absorción a través de la mucosa nasal. Si la CD se incorpora en proporciones más 
elevadas (5-10\%), se forman complejos estables que difunden con dificultad con lo que la absorción nasal disminuye [47]. La incidencia de otros mecanismos, como el impedimento estérico o la interacción de la CD o el fármaco con otros componentes de la formulación, explican los resultados aparentemente contradictorios recogidos en la bibliografía $[48,49]$.

Como regla general, si se forma un complejo estable entre un fármaco hidrofóbico y la $\mathrm{CD}$, la velocidad de difusión dentro del entramado es menor que la que presenta el fármaco libre [50,51]. La aplicación de modelos matemáticos que permiten estimar la difusividad del fármaco libre y del complejo CD-fármaco en sistemas ternarios fármaco/CD/polímero, pone de manifiesto una progresiva reducción de la velocidad de cesión del fármaco a medida que se incrementa la proporción de CD [52]. Esta tendencia no se manifiesta en las dos situaciones siguientes:

a) cuando la formación del complejo incrementa significativamente la solubilidad y facilita la disolución y la salida del fármaco del entramado polimérico [53]. En este caso, un elevado gradiente de concentración de fármaco permite mejorar significativamente la absorción oral y transdérmica [54]. Especialmente ilustrativos son los resultados obtenidos con HP- $\beta$-CD y $\gamma$-CD y con ibuprofeno, ketoprofeno y prednisolona en hidrogeles de polivinilpirrolidona (PVP) reticulada con ácido polietilenglicoldimetacrilico [55]. La incorporación de los fármacos se llevó a cabo sumergiendo hidrogeles secos en una disolución saturada de fármaco (hidrogeles control) o en una disolución de complejo CD-fármaco. La relación molar CD:fármaco en la disolución de carga varió entre 6 y 50, solubilizándose la totalidad de la dosis al encontrarse la CD en exceso. Con HP- $\beta$-CD se consiguió incrementar, con respecto al hidrogel control, 6, 9 y 3 veces la carga de ibuprofeno, ketoprofeno y prednisolona. Con $\gamma$-CD se duplicó la cantidad de prednisolona cargada, pero no se mejoró la carga de ibuprofeno o ketoprofeno debido a la baja solubilidad de los complejos. La HP-B-CD incrementó la velocidad de cesión de los tres fármacos, mientras que la $\gamma-\mathrm{CD}$ no solo no alteró la liberación de ibuprofeno, que tiene una baja tendencia a formar complejos, 
sino que retrasó la cesión de ketoprofeno y de prednisolona. Este último efecto se explica por el gran tamaño y el bajo coeficiente de difusion de los complejos que forma la $\gamma$-CD. Si la constante de afinidad CD-fármaco es suficientemente alta, no se necesario preparar previamente el complejo ya que este puede formarse de forma espontánea una vez que se hidrata el entramado [49].

b) cuando el fármaco interacciona fuertemente con el entramado polimérico, como ocurre en el caso del hidrocloruro de propanolol y el ácido poliacrílico (Carbopol ${ }^{\circledR}$ ). El fármaco, catiónico, forma agregados insolubles con las cadenas poliméricas y reduce de manera significativa el grado de hinchamiento y la bioadhesividad de los microgeles. La complejación con $\beta$-CD minimiza las interacciones fármaco-polímero, devolviendo a los microgeles su comportamiento característico e incrementando la velocidad de cesión [56].

Cuando no se forman complejos, las CDs hidrosolubles pueden aumentar la velocidad de cesión formando, a medida que se disuelven y abandonan la matriz del hidrogel, canales por los que puede salir el fármaco. Las CDs menos hidrofílicas aumentan la tortuosidad, dificultan la difusión y retrasan la cesión [46]. Una buena prueba de la complejidad de los efectos de las CDs sobre la cesión de fármaco a partir de entramados físicamente reticulados, es el comportamiento que se observó al incorporar $\beta-C D$ e HP- $\beta-C D$ a geles y comprimidos matriciales de hidroxipropilmetilcelulosa (HPMC K4M) junto con un fármaco hidrosoluble (diclofenaco sódico) o un fármaco de reducida solubilidad (sulfametizol). Los dos fármacos forman complejos de constantes de estabilidad de 100.6 y $115.2 \mathrm{M}^{-1}$ (diclofenaco sódico) y 651.8 y $563.9 \mathrm{M}^{-1}$ (sulfametizol) con $\beta-C D$ y HP- $\beta-C D$, respectivamente $[57,58]$. En geles preparados con HPMC al 2\%, una relación molar CD:fármaco 0.5:1 dio lugar a un incremento en la velocidad de difusión al minimizarse las interacciones hidrofóbicas entre el polímero y el fármaco. En cambio, un exceso de CD, en especial de la variedad más voluminosa (HP- $\beta$-CD), dificultó la difusión de los complejos a través de la malla relativamente estrecha del entramado. En comprimidos matriciales preparados con relaciones 
CD/lactosa elevadas se observó un incremento marcado de la velocidad de cesión de sulfametizol, mientras que la cesión de diclofenaco sódico se ralentizó, lo que prueba que el predominio de uno u otro efecto depende de la hidrofília del fármaco [59].

Otro aspecto a tener en cuenta es la posibilidad de que se produzca una complejación parcial de algún componente polimérico con la CD, en particular de un copolímero anfifílico o de un polímero con grupos hidrofóbicos [60]. Por ejemplo, las CDs se pueden utilizar para modular la viscosidad y la respuesta a la luz de polímeros funcionalizados con grupos azobenceno, que experimentan cambios de conformación trans/ cis cuando se irradian con luz UV. En la oscuridad los isómeros trans, hidrofóbicos, se asocian y actúan como puntos de unión a lo largo de la cadena polimérica, con lo que aumenta la viscosidad del gel. Bajo irradiación, la transición de la forma trans a cis (más hidrofílica) hace que las uniones se rompan. El isómero trans del azobenceno puede formar complejos con $\alpha-\mathrm{CD}$, inhibiendo su autoasociación e incluso revirtiendo el efecto de la radiación UV sobre la viscosidad [61]. A su vez, el isómero cis se puede complejar con HP- $\beta$-CD. Cuando los sustituyentes azobenceno están anclados sobre las cadenas de un copolímero anfifílico, como el poli(N,N-dimetilacrilamida-co-metacriloiloxiazobenceno) (DMA-MOAB), y se añade HP- $\beta-C D$, su capacidad para interaccionar con otros copolímeros anfifílicos y dar lugar a micelas se puede alterar considerablemente modificando las condiciones de irradiación. Este fenómeno puede ser útil para modular la difusión de solutos hidrofílicos a través de hidrogeles [62].

Las CDs también pueden formar complejos de estequiometria muy superior a 1:1 con copolímeros bloque, dando lugar a estructuras en forma de collar denominadas polipseudorotaxanos [63]. Las CDs se ensartan en las cadenas de polímero acumulándose en las regiones más favorables para la complejación (por ejemplo, en los bloques polióxido de etileno la $\alpha-C D$ o en los bloques polióxido de propileno la $\beta$-CD) [64-66]. Si los extremos del polímero se bloquean con grupos voluminosos de manera que las CDs no puedan abandonar el polímero, se obtienen estructuras conocidas como polirotaxanos. Las interacciones intermoleculares entre unidades de CD incorporadas a polirotaxanos pueden dar lugar a la formación 
de superestructuras en forma de nanotubos. También se ha propuesto la reticulación de las CDs de polirotaxanos adyacentes [67] (Figura 1.4b) y para unir químicamente las CDs que ocupan los extremos de las cadenas poliméricas [68] con el fin de obtener geles con puntos de reticulación deslizantes. Un diseño adecuado de los polirotaxanos permite una amplia modulación de las propiedades del hidrogel, lo que abre interesantes posibilidades en el ámbito biomedicina $[63,69,70]$. Aunque el número de estudios sobre las posibilidades que ofrecen los polirotaxanos para incrementar la solubilidad y modificar la difusividad de los fármacos es todavía reducido, los resultados de los que se dispone indican que la complejación espontánea de copolímeros bloque con CDs eleva la concentración crítica micelar del copolímero y reduce el número de micelas y de cavidades de CD libres para albergar moléculas de fármaco [71-73]. Esto hace que la eficacia de solubilización de fármacos hidrofóbicos se reduzca. Además, en el caso de los copolímeros bloques de óxido de polietileno/óxido de polipropileno sensibles a cambios de temperatura, como los PEO-PPOPEO (poloxamer o Pluronic ${ }^{\circledR}$ ), se producen modificaciones importantes en la viscoelasticidad de los geles y en la temperatura de transición solgel $[72,74,75]$. La adición de HP- $\beta$-CD o M- $\beta$-CD $(5 \% \mathrm{p} / \mathrm{v})$ a dispersiones de Pluronic F127 (15\% p/v) da lugar a un incremento de 5 y $15^{\circ} \mathrm{C}$, respectivamente, de la temperatura de gelificación y reduce marcadamente el módulo elástico o de almacenamiento $\left(G^{\prime}\right)$ y el módulo viscoso o pérdida (G“'). Además, el Pluronic F127 desplaza fácilmente las moléculas huésped de la cavidad de las CDs, aumentando la proporción de fármaco libre en el medio [76]. Estos hechos llaman la atención sobre la necesidad de identificar la naturaleza y la estequiometria de los complejos cuando se preparan sistemas ternarios fármaco-polímero-CD, una práctica habitual en tecnología farmacéutica.

Un paso adelante en el campo de los sistemas ternarios es la preparación de geles estables usando un mecanismo "llave-cerradura" o "cremallera", en el que CDs unidas covalentemente a la cadena polimérica reconocen ciertos grupos de otros polímeros, originando un entramado tridimensional (Figura 1.4c). La reticulación mediada por CD da lugar a entramados que exhiben propiedades intermedias entre 
las de los hidrogeles físicos, en los que las interacciones son reversibles, y las de los hidrogeles reticulados químicamente que son estables frente a la dilución. Este fenómeno se ha observado al mezclar: i) un polímero con CDs "colgantes" con un polímero con cadenas laterales hidrofóbicas de 4-tert-butilanilida [77]; ii) un conjugado de quitosanoCD con quitosano con grupos adamantilo o polietilenglicol [78]; iii) poli(acrilamida)-CD con poli(acrilamida) con anillos aromáticos [79]; y iv) polímeros de $\beta$-CD con poli( $\mathrm{N}$-isopropilacrilamida) con grupos adamantilo o dodecilo [80]. Por ejemplo, se produce un autoensamblaje espontáneo entre polímeros de $\beta$-CD (poli- $\beta$-CD) y dextranos con cadenas alquílicas o grupos adamantilo colgantes (Figura 1.5). Cuando se mezclan disoluciones acuosas de ambos polímeros en concentraciones de $6.6-7.5 \% \mathrm{p} / \mathrm{p}$ se observa una inmediata separación de fases. La fase gel presenta una alta concentración de ambos polímeros, con valores de G' y G“' 400-500 Pa y 1200-1400 Pa, respectivamente [81,82]. Si las concentraciones de polímeros son más bajas $(0.1-1 \% \mathrm{p} / \mathrm{p})$ se forman partículas nanométricas de tamaño variable según el grado de sustitución del polímero con grupos que forman complejos con las CDs [83,84]. Si los polímeros cuentan con grupos ionizables, se pueden conseguir geles de viscosidad variable en función del $\mathrm{pH}$ del medio. Los hidrogeles se pueden cargar con moléculas que formen complejos con poli- $\beta-C D$, incorporándolas a sus disoluciones antes de mezclar con la disolución de dextrano (Figura 1.5). Las cavidades que no contengan fármaco estarán disponibles para albergar las cadenas alquílicas y actuar como puntos de unión entre ellas. Estos geles proporcionan perfiles de liberación sostenida de benzofenona y tamoxifeno durante más una semana. Además, la reversibilidad de la complejación polímero-CD hace posible la administración del gel mediante inyección a través de agujas relativamente finas. Una ligera presión causa la decomplejación y un descenso de la viscosidad, y el sistema fluye fácilmente. Al cesar la presión, los geles recuperan rápidamente los valores iniciales de $\mathrm{G}^{\prime} \mathrm{y}$ G' [81]. Estas propiedades junto con una excelente biocompatibilidad permiten augurar a estos sistemas de gelificación in situ un futuro muy prometedor en el campo de la biomedicina. 
a

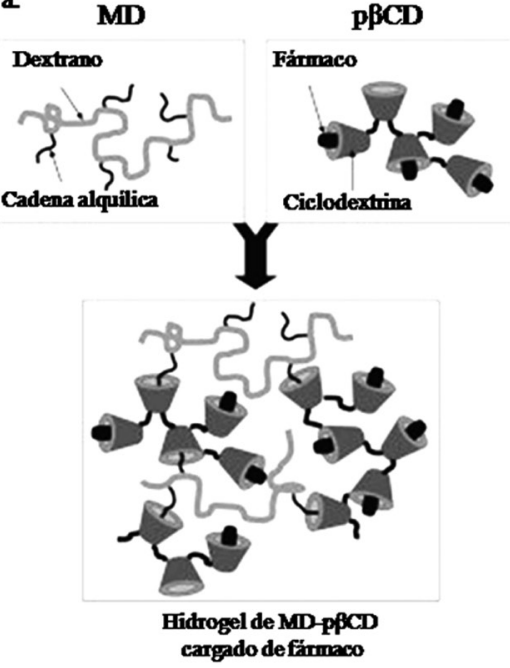

b.
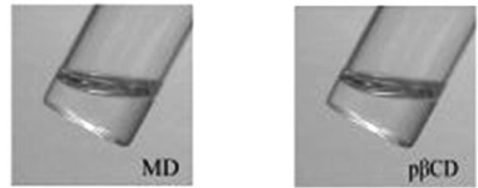

$\mathrm{PBCD}$

Figura 1.5. (a) Interacción espontánea de un dextrano alquil-modificado (MD) con poli- $\beta$-CD para dar lugar a un gel. Algunas cadenas alquílicas del MD forman complejos con las CDs del poli- $\beta$-CD. Las CDs libres pueden formar complejos de inclusión con fármacos hidrofóbicos. (b) Aspecto de las disoluciones de MD y da poli- $\beta$-CD y de la mezcla de ambas transcurridos 5 segundos. Adaptado de referencia [81] con permiso de John Wiley and Sons.

\subsection{Hidrogeles con CD integradas en su estructura}

Se pueden preparar hidrogeles químicamente reticulados con CDs integradas en el esqueleto polimérico, acudiendo a alguno de los procedimientos siguientes: a) reticulación directa de las CDs (condensación con un agente reticulante), b) copolimerización de las CDs con comonómeros acrílicos o vinílicos, y c) anclaje de las CDs a entramados preformados. Cuando los entramados entran en contacto con un medio acuoso, las CDs no se diluyen, a diferencia de lo que ocurre con las disoluciones de CDs individuales y con los hidrogeles reticulados físicamente. El volumen de agua que puede entrar en un hidrogel reticulado químicamente está limitado por el propio entramado y, dado que las CDs se encuentran covalentemente unidas, el hidrogel hincha sin disolverse y sin perder componentes. Esto genera un microambiente rico en CD con cavidades disponibles para 
interaccionar con moléculas de fármaco. En estas estructuras, la afinidad CD-fármaco se convierte en el principal factor responsable del control de la liberación. Cuando una molécula de fármaco se decompleja de una cavidad de $\mathrm{CD}$, encuentra en su entorno otras cavidades disponibles para formar un nuevo complejo. El desplazamiento del fármaco a través del hidrogel será más o menos rápido dependiendo del grado de ocupación de las cavidades del entramado y de su afinidad por ellas (Figura 1.6). A medida que avanza el proceso de cesión, el número de cavidades disponibles para formar complejos con el fármaco se va incrementando. Ello ralentiza la cesión e incluso hace posible que algunas moléculas que se habían liberado previamente puedan ser recaptadas si permanecen en torno al hidrogel. En consecuencia, los hidrogeles de CD poseen características únicas para retener fármacos y pueden resultar muy útiles en el desarrollo de sistemas de liberación controlada. Es importante destacar que las CD covalentemente unidas al entramado no ven reducida su capacidad para formar complejos, sino que incluso se puede incrementar, en especial con compuestos de gran tamaño molecular que requieren varias unidades de CD por molécula para formar el complejo [85-91]. En las secciones siguientes se describen las estrategias a seguir para el desarrollo de entramados de CDs reticuladas.

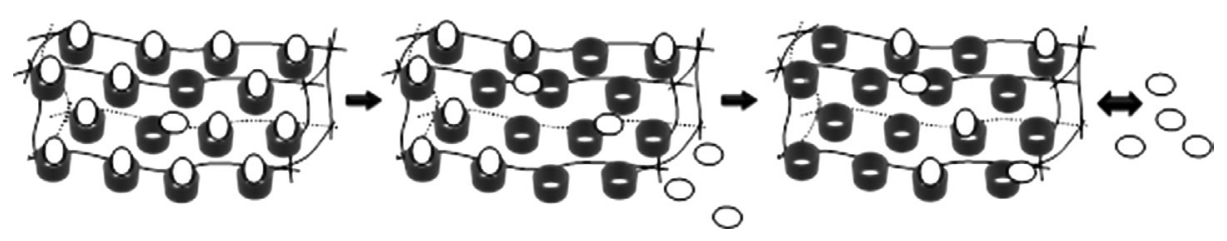

Figura 1.6. Liberación de un fármaco a partir de un hidrogel de CDs químicamente reticuladas. El fármaco va ocupando cavidades sucesivas hasta llegar a la superficie del entramado.

\subsubsection{Reticulación directa}

Los primeros polímeros e hidrogeles de CDs se prepararon por reacciones de condensación de los grupos hidroxilo de CDs naturales o de 
los grupos amino o ácido carboxílico de CDs funcionalizadas, utilizando agentes reticulantes di- o multifuncionales tipo aldehído, cetona, isocianato o epóxido (epichlorhidrina) [92]. Aunque la reacción transcurre de manera espontánea, normalmente se incorpora un catalizador para incrementar la velocidad del proceso [93]. El agente reticulante más utilizado es la epiclorhidrina (EPI). En medio alcalino, sus dos grupos funcionales pueden reaccionar entre sí o con grupos hidroxilo de las CDs, para dar lugar a una mezcla de CDs reticuladas unidas por cadenas cortas de EPI polimerizada (Figura 1.7) [94,95]. Los hidrogeles de EPI-CD (generalmente microgeles) pueden hinchar en medio acuoso. Controlando la reacción (por ejemplo, parando la reticulación en una determinada etapa) es posible obtener polímeros de CD hidrosolubles [88]. La relación EPI: $\beta$-CD determina la proporción de cavidades de CD disponibles para interaccionar con el fármaco; alcanzándose el máximo con hidrogeles que contienen un $50 \%$ de $\beta$-CD [96].

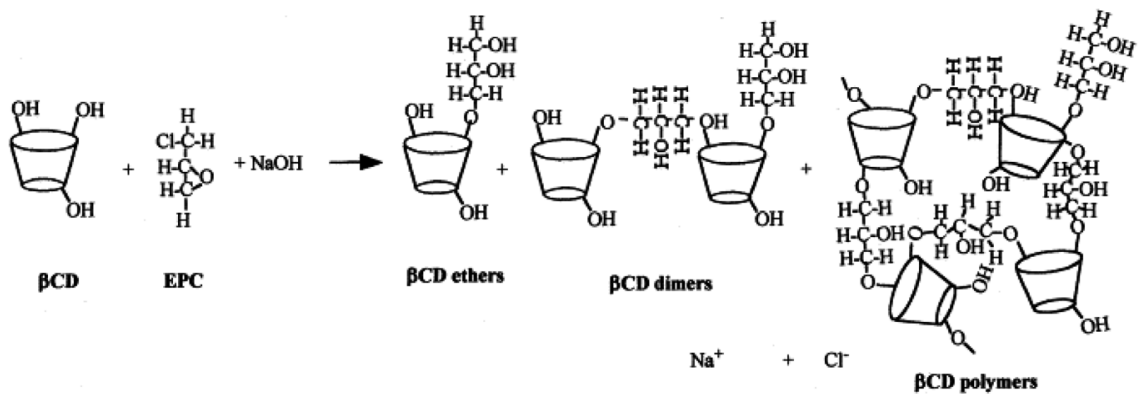

Figura 1.7. Hidrogel de EPI-CD. Reproducido de referencia [94] con permiso de Elsevier.

Los microgeles de EPI-CD se han evaluado como adsorbentes para extraer fármacos y moléculas hidrofóbicas del agua [97-99], para separar compuestos en matrices complejas [100-103] o en cromatografía [104,105] y para separar enantiómeros a partir de mezclas racémicas [106-108]. Las propiedades mecánicas de los microgeles se pueden modular incorporando polímeros hidrofílicos (por ejemplo, PVA) y otros agentes 
reticulantes [109, 110]. También se les puede dotar de sensibilidad a cambios de temperatura, uniendo poli(N-isopropilacrilamida) (PNIPA) a $\beta$-CDs previamente reticuladas [111] o preparando entramados interpenetrados (IPN) o semiinterpenetrados (semi-IPN) de EPI-CD y PNIPA [112]. Estos sistemas permiten regular la velocidad de cesión de los fármacos en función no sólo de la afinidad por las CDs sino también de las condiciones de temperatura de su entorno [112, 113]. También se han preparado microgeles sensibles a cambios de $\mathrm{pH}$, interpenetrando microgeles de EPI-CD-PVA con poli(ácido metacrílico) (PMAA) [114]. Por otra parte, el elevado número de grupos hidroxilo de las CDs las hace muy útiles para desarrollar entramados EPI-CD sensibles a campos eléctricos. Estos materiales inteligentes experimentan cambios rápidos y reversibles en sus propiedades reológicas bajo la acción de pequeños campos eléctricos; sin embargo, no resisten un fuerte campo eléctrico durante un tiempo prolongado y la polarización se ve limitada por la rigidez y la alta densidad de CDs [115]. La co-reticulación con almidón permite obtener entramados que, mezclados con aceite de silicona, presentan unas buenas propiedades electroreológicas [116].

Algunos hidrogeles de EPI-CD muestran una elevada capacidad de retención de solutos debido, no sólo a la formación de complejos de inclusión, sino también al establecimiento de interacciones específicas con los numerosos grupos hidroxilo de las CD. Sacando partido de este mecanismo se han desarrollado hidrogeles selectivos para creatinina [117]. Los hidrogeles se preparan a $\mathrm{pH}$ alcalino dado que en estas condiciones los OH-6 están ionizados y pueden interaccionar electrostáticamente con los grupos amino de la creatinina. Una vez formado el hidrogel y eliminada la creatinina, se mantiene la conformación de las CDs, lo que permite la recaptación selectiva de creatinina de medios acuosos. La máxima selectividad se manifiesta con proporciones molares $\beta$-CD:creatinina 3:2 y $\beta$-CD-EPI 1:10. Los entramados de EPI-CD también se pueden funcionalizar con grupos amonio cuaternario para que actuen como trampas de sales biliares [118].

La utilización de diisocianatos como agentes reticulantes también permite elaborar macro y microhidrogeles de CDs (Figura 1.8) [119-121]. 
Los hidrogeles de $\beta$-CD y diaminopoli(etilenglicol), reticulados con hexametilendiisocianato son muy hidrofílicos, biocompatibles y capaces de cargar y ceder de forma controlada estradiol, quinina o lisozima [122].

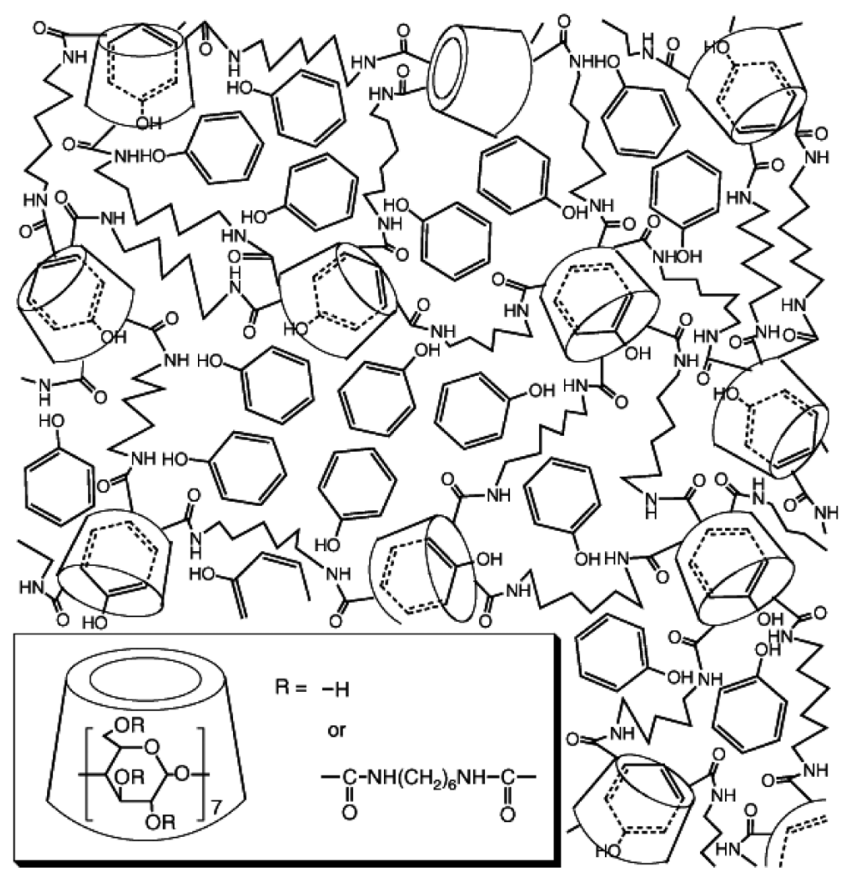

Figura 1.8. Estructura de un entramado de $\beta$-CDs reticuladas con hexametilendiisocianato en proporción 1:8 y formación de complejos de inclusión con fenol. Reproducido de referencia [120] con permiso de John Wiley and Sons.

Los diisocianatos también se han empleado para obtener polímeros hidrofílicos de CDs hiperramificados con capacidad para formar complejos [123] y partículas nanoporosas de CDs para captar solutos de medios acuosos y cederlos a fases orgánicas [124]. La combinación de esta aproximación con la tecnología de moldeado molecular (molecular imprinting) permite mejorar la selectividad de la carga y conseguir un mejor control de la cesión [125]. El grupo de Asanuma y Komiyama ha evaluado ampliamente las posibilidades que ofrece el moldeado molecular, para potenciar la capacidad de los entramados de $\beta$-CD reticulados con tolueno-2,4-diisocianato en la separación 
selectiva de moléculas con actividad biológica y en la extracción de contaminantes de efluentes líquidos [126]. La reticulación en presencia de colesterol o estigmasterol conduce a la formación de entramados en los que dímeros o trímeros de $\beta-C D$ actúan cooperativamente para atrapar cooperativamente moléculas esteroídicas de gran tamaño. Una vez completada la polimerización y retiradas las moléculas que han servido como moldes, los hidrogeles imprinted (MIP) pueden captar de un medio acuoso colesterol y estigmasterol, mostrando una afinidad mucho menor por otras estructuras químicamente relacionadas (Figura 1.9) $[127,128]$. En general, la reticulación de $\beta$-CD con diisocianatos conduce a la obtención de hidrogeles con menor tamaño de malla y grado de hinchamiento, en comparación con los obtenidos con EPI, y en los que las interacciones hidrofóbicas inespecificas se producen con más facilidad $[129,130]$.
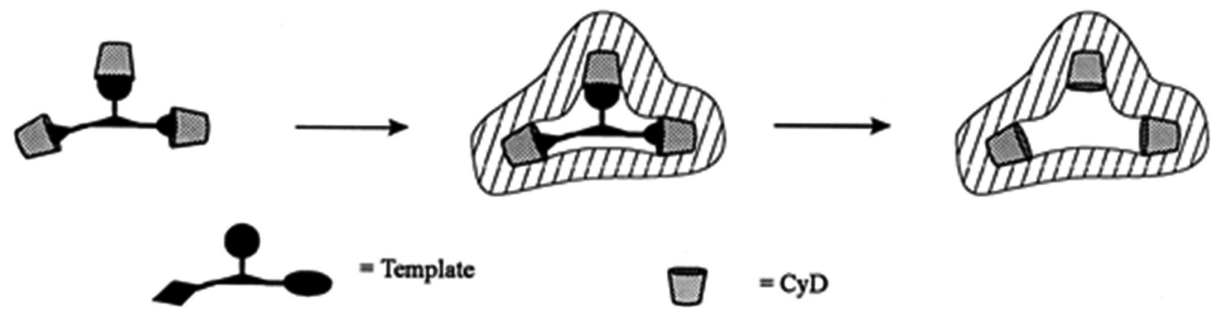

Figura 1.9. Esquema de un MIP de CD obtenido utilizando diisociantos como agentes reticulantes. Reproducido de referencia [127] con permiso de la American Chemical Society.

Para desarrollar microcápsulas recubiertas con $\beta$-CD reticuladas se ha utilizado una técnica de emulsión-polimerización con cloruro de diacilo [131]. Las moléculas huésped acceden rápidamente a las cavidades de las CDs, completandose la carga en pocos minutos. Las microcápsulas sostienen la cesión de propanolol durante varias horas [132]. Los reticulantes con grupos carbonilo activos también se han aplicado a la obtención de nanoesponjas de 350-600 nm de diámetro y grados de prorosidad variables, potencialmente útiles como transportadores de fármacos muy diversos [133-134]. Por su parte, la condensación con ácidos policarboxílicos, como ácido cítrico, ácido 1,2,3,4-butanotetra- 
carboxilico o poli(ácido acrílico) (PAA) (Figura 1.10), constituye una aproximación limpia para obtener de entramados de $\mathrm{CD}$, aunque tiene el inconveniente de que hay que eliminar el agua que se genera en la reacción de esterificación, aplicando vacío o temperaturas superiores a $140^{\circ} \mathrm{C}[135]$.

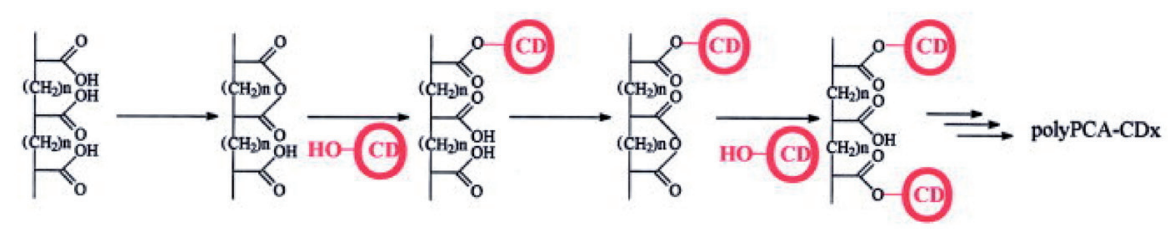

Figura 1.10. Estructura de un entramado de CDs reticuladas por condensación con poli(ácido carboxílico)s. Reproducido de referencia [135] con permiso de John Wiley and Sons.

Por último, la capacidad de las CDs para reaccionar con grupos epóxido en condiciones suaves ha llevado a desarrollar procedimientos que permiten unir las CDs en un solo paso [136]. El etilenglicoldiglicidileter (EGDE) cuenta en su estructura con dos grupos epoxido de reactividad similar, capaces de reaccionar simultáneamente con los grupos hidroxilo de una $\mathrm{CD}$ o de un polisacárido lineal, dando lugar a hidrogeles viscoelásticos de elevada biocompatibilidad [7,136-138]. Para que se formen hidrogeles de HP- $\beta$-CD se requiere como mínimo un $10 \%$ p/p de HP- $\beta$-CD y un $14.28 \%$ p/p de EGDE. Estas proporciones permiten que $2 / 3$ de los grupos hidroxilo de cada $C D$ reaccionen con el agente reticulante. Los hidrogeles de HP- $\beta$-CD y de M- $\beta$-CD se comportan con superabsorbentes (incorporan hasta $1000 \% \mathrm{p} / \mathrm{p}$ de agua) y pueden incorporar cantidades de diclofenaco sódico y de estradiol de 2 a 500 veces superiores a las que se conseguirían si el fármaco se alojase únicamente en la fase acuosa del hidrogel. La afinidad del entramado por los fármacos durante la etapa de incorporación como el control de cesión al entrar en contacto con medio acuoso muestran una estrecha correlación con el valor de la constante de complejación fármaco-CD [139] (Figura 1.11). 

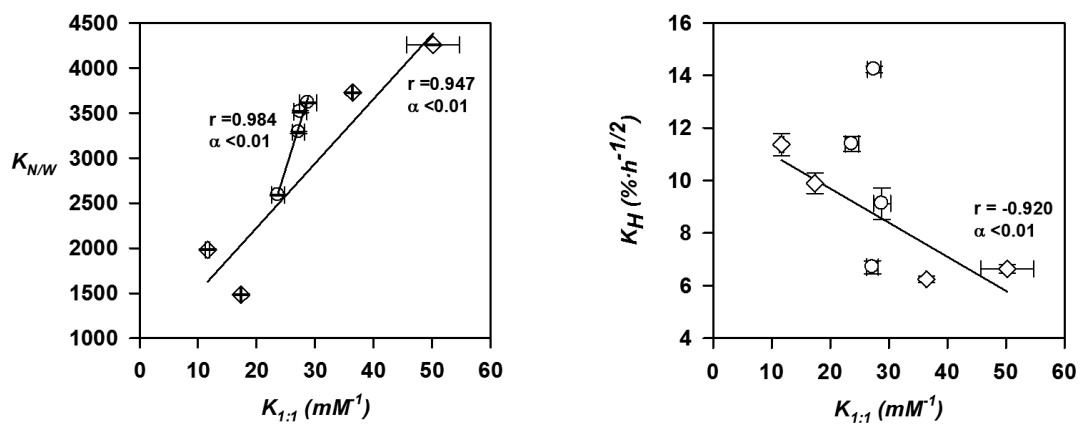

Figura 1.11. Dependencia del coeficiente de reparto entramado/agua $\left(\mathrm{K}_{\mathrm{N} / \mathrm{W}}\right)$ y de la constante de liberación $\left(\mathrm{K}_{\mathrm{H}}\right)$ del estradiol a partir de hidrogeles de HP- $\beta$-CD $(\diamond)$ y M- $\beta$-CD (o) respecto de las constantes de estabilidad de los complejos $\left(\mathrm{K}_{1: 1}\right)$ [139].

En el caso del estradiol, se consiguieron perfiles de cesión sostenida durante una semana [139]. La carga y la liberación de fármaco están controladas principalmente por la constante de afinidad fármaco/CD, y la incorporación de los éteres de celulosa dota a los hidrogeles de mayor flexibilidad y acelera ligeramente el proceso de cesión (Figura 1.12).

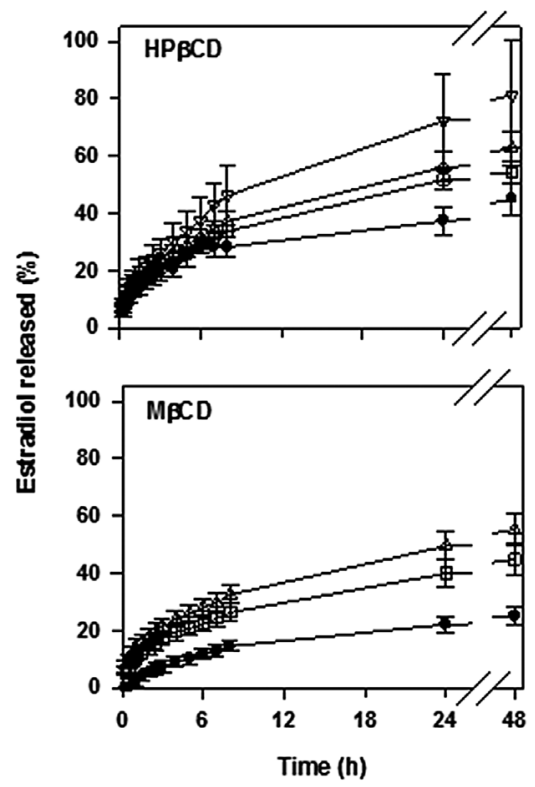

Figura 1.12. Cesión del estradiol a partir de hidrogeles de HP- $\beta$-CD or M- $\beta$-CD preparados con $20 \%$ de CD $(\bullet), 20 \%$ CD - 0.25\% HPMC ( $\square), 25 \%$ CD - 0.25\% HPMC $(\Delta)$, y $30 \%$ CD $-0.25 \%$ HPMC $(\nabla)$. Reproducido de referencia [139] con permiso de Elsevier. 
El procedimiento de reticulación con EGDE permite obtener macrogeles y nanogeles mixtos de $\mathrm{HP}-\beta-\mathrm{CD}$ o $\gamma$-CD y diversos polímeros relacionados estructuralmente, como HPMC, metilcelulosa (MC), hidroxipropilcelulosa (HPC), carboximetilcelulosa sódica (CMCNa), dextrano o agar-agar [140142]. Estos hidrogeles presentan un microambiente rico en cavidades de CD muy adecuado para incorporar eficazmente fármacos antifúngicos como el sertaconazol [140], antibióticos como el ciprofloxacino [141], o antiinflamatorios como la dexametasona [142]. En general, la aplicación de una etapa de calefacción en autoclave $\left(121^{\circ} \mathrm{C}, 20 \mathrm{~min}\right)$ promueve la incorporación del fármaco sin afectar a las propiedades mecánicas del hidrogel.

Aplicando un procedimiento similar, se prepararon hidrogeles de HP- $\beta$ CD con dominios interpenetrados de poli(ácido acrílico) (PAA, Carbopol®) con el objetivo de combinar la capacidad de respuesta frente a cambios de $\mathrm{pH}$ y las propiedades mucoadhesivas del Carbopol con la capacidad de formación de complejos de las CDs reticuladas [143]. Estos hidrogeles presentan un entramado contínuo de CDs y dominios discontínuos de carbopol, resultando en un IPN a microescala (ms-IPN). Estos ms-IPNs tienen las siguientes ventajas: i) para su obtención no se requiere la preparación previa de monómeros acrílicos de CD; ii) cuando se hidratan no pierden componentes, como ocurre con los semi-IPNs convencionales; iii) la presencia de PAA reticulado comunica bioadhesividad y capacidad de respuesta a cambios de pH (Figura 1.13); iv) la estructura descontinúa puede facilitar la movilidad de los entramados lo que los dota de excelentes propiedades mecánicas; y v) el producto final se obtiene en un solo paso.

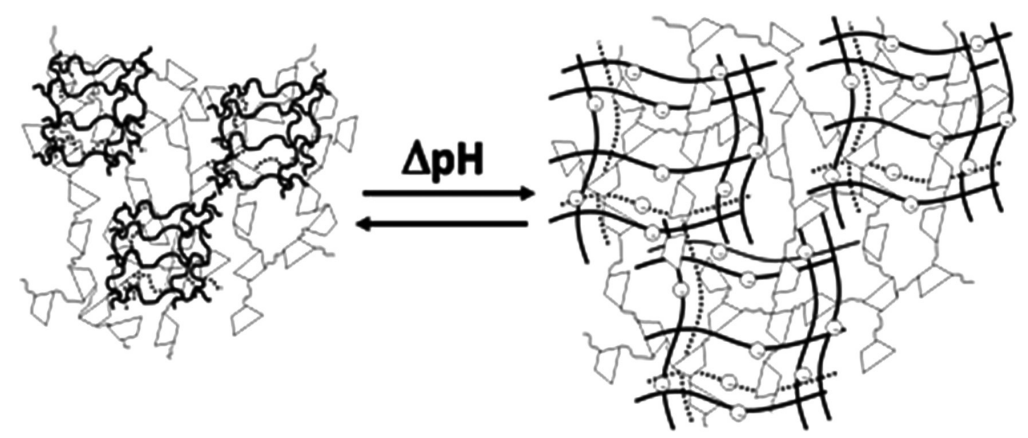

Figura 1.13. Hinchamiento de un ms-IPN de HP- $\beta$-CD y carbopol en respuesta a cambios de $\mathrm{pH}$. Reproducido de referencia [143] con permiso de Elsevier. 
Los ms-IPNs han mostrado un gran potencial como vehículos de estradiol y ketaconazol, incorporando hasta 2000 veces más fármaco que el que se puede disolver en la fase acuosa [143]. El carbopol contribuye a incrementar la capacidad de incorporación de fármaco haciendo que, a pH neutro, los entramados tengan una malla más abierta, a través de la que el fármaco difunde con mayor facilidad y puede entrar en contacto más fácilmente con las CDs para formar complejos. Estos IPNs sostienen la cesión durante varios días, a una velocidad que depende del $\mathrm{pH}$ y que se puede modular modificando la proporción de carbopol (Figura 1.14).

\subsubsection{Hidrogeles preparados por copolimerización de monómeros de CD}

Una alternativa a la reticulación directa de las CDs consiste en preparar monómeros de CD (Figura 1.15) que puedan copolimerizar con los monómeros vinílicos o acrílicos utilizados habitualmente en la preparación de hidrogeles. La mayoría de las síntesis de monómeros de CD se centran en los grupos hidroxilo del anillo glucopiranósico. Teniendo en cuenta que las CDs presentan 18 ( $\alpha$-CD), 21 ( $\beta$-CD) ó 24 ( $\gamma-C D)$ grupos hidroxilo susceptibles de ser sustituidos, el número de derivados posibles es muy elevado. Por otra parte, el hecho de que un elevado número de grupos hidroxilo tenga una reactividad similar, hace que la preparación de monómeros monofuncionalizados resulte difícil.

Se pueden preparar monómeros monofuncionalizados haciendo reaccionar $\alpha$-CD o $\beta$-CD con un éster de m-nitrofenilo en medio alcalino (Figura 1.15a). Los ésteres de nitrofenilo forman complejos con las CDs y provocan una transesterificación selectiva de uno de los grupos hidroxilo secundarios, reduciendo las posibilidades de formar derivados multifuncionales $[144,145]$. La copolimerización de acriloil- $\beta$-CD con $\mathrm{N}$-isopropilacrilamida (NIPA) da lugar a hidrogeles porosos que experimentan rápidas transiciones de fase en medio acuoso [146]. Además, acriloil- $\alpha-C D$ y acriloil-(6-O- $\alpha-D-g l u c o s i l)-\beta-C D$ resultan útiles para preparar partículas imprinted con afinidad por moléculas que pueden formar complejos con varias CDs simultáneaments, como es el caso de 
la vancomicina, cefazolina, feneticilina y algunos dipéptidos [147]. La combinación de bisacriloil- $\beta$-CD y monómeros iónicos (ácido sulfónico 2-acriloilamido-2,2'-dimetilpropano) conduce a entramados con gran afinidad por moléculas anfifílicas como la fenilalanina, discriminando incluso sus enantiómeros [148,149].

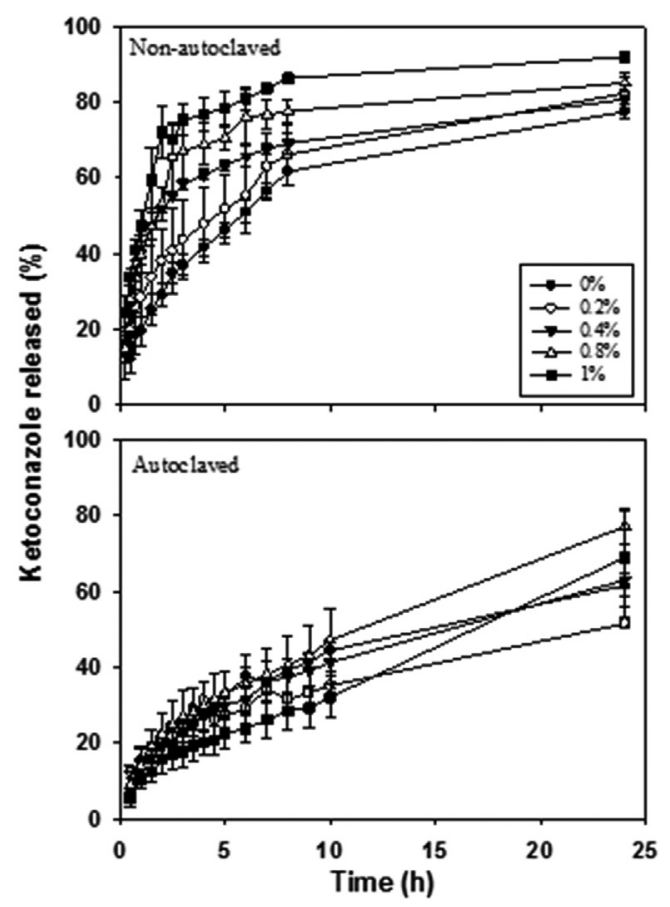

Figura 1.14. Perfiles de cesión de ketoconazol, en una disolución de dodecilsulfato sódico al $0.3 \%$ ( $\mathrm{pH} 7.8$ ), a partir de hidrogeles HP- $\beta$-CD/carbopol preparados con distintas proporciones de carbopol. Reproducido de referencia [143] con permiso de Elsevier. 


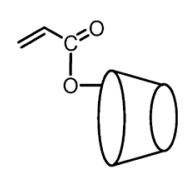

(a) Acriloil-CD

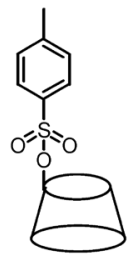

(b) 6-O-(p-tosil)-CD

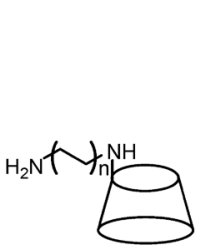

(c) 6-(alquilenodiamino)-CD

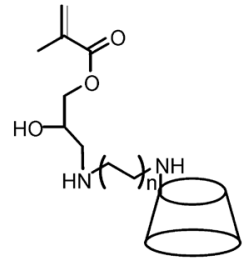

(d) GMA-(alquilenodiamino)-CD

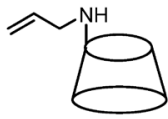

(e) Mono-(6-N-alilamino-6-deoxi)-CD

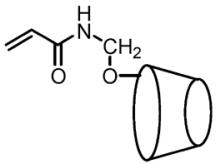

(f) Acrilamidometil-CD

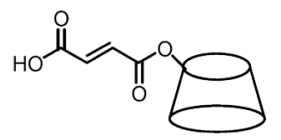

(g) Anhidrido maléico (MAH)-CD

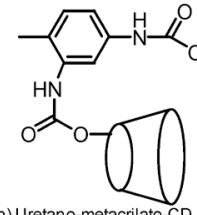

(h) Uretano-metacrilato-CD

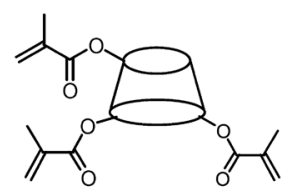

(i) 2,3-di-O-metacrilato-6-metacrilato-CD

Figura 1.15. Estructura de algunos monómeros de ciclodextrina que se han ensayado como componentes de entramados poliméricos.

Los derivados monotosilo de la $\beta$-CD (Figura 1.15b) se pueden obtener haciendo reaccionar un grupo hidroxilo C6 primario con el cloruro de tosilo [150-152]. Para preparar nuevos monómeros monofuncionalizados con grupos amino primario, se hace reaccionar etilenodiamina (EDA) o 1,6-hexanodiamina (HAD) con mono-6-Ots- $\beta$-CD [153]. A continuación, el grupo amino reacciona con glicidilmetacrilato (GMA) y se obtienen GMA-EDA- $\beta$-CD y GMA-HAD- $\beta$-CD, que son monómeros monometacrilato de $\beta$-CD (Figura 1.16). 

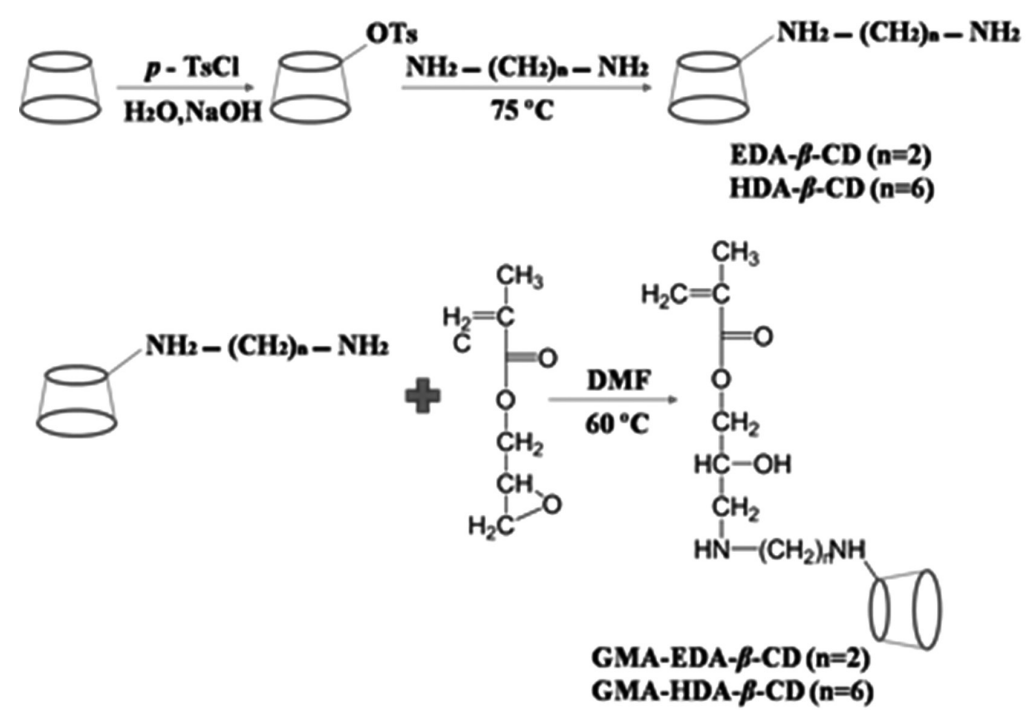

Figura 1.16. Ruta de síntesis de monómeros monometacrilato de $\beta$-CD (GMAEDA- $\beta$-CD y GMA-HDA- $\beta$-CD) a partir de mono-6-OTs- $\beta$-CD y etilenodiamina (EDA) ó 1,6-hexanediamina (HAD). Reproducido de referencia [153] con permiso de John Wiley and Sons.

Los monómeros derivados de la acrilamida, como la acrilamidometil-CD (Figura 1.15f) [154], combinados con acrilato sódico se han mostrado útiles para preparar hidrogeles sensibles a cambios de $\mathrm{pH}$, que regulan la carga y cesión de fármaco por un doble mecanismo de afinidad por las CDs y de respuesta del entramado al pH [155]. Estos monómeros también se han utilizado para peparar entramados capaces de captar selectivamente aminoácidos u oligopéptidos en medio acuoso [156-158].

La condensación de CDs con anhidrido maleico (MAH) conduce a la formación de monómeros que combinan la capacidad complejante de las CDs con la capacidad de respuesta frente a cambios de $\mathrm{pH}$ de los grupos ácido carboxílicos (Figura 1.15g) [159]. Los hidrogeles de PNIPA (91-64\% p/p)-co-MAH- $\beta$-CD (9-36\% p/p) responden a estímulos térmicos, al $\mathrm{pH}$ y a la fuerza iónica y se pueden utilizar para cargar clorambucilo y cederlo a distinta velocidad en función del pH del medio [160]. Los cambios en el grado de hinchamiento son reversibles y reproducibles 
después de varios ciclos [159] (Figura 1.17). MAH- $\beta$-CD también se puede copolimerizar con copolímeros bloque sensibles a cambios de temperatura, como los poloxamer o Pluronic ${ }^{\circledR}$ [161], o biodegradables como el poli(D,L-ácido láctico) [162].

Para preparar monómeros multifuncionales de uretano-metacrilato- $\beta$ CD (Figura 1.15h) se suele acudir a un procedimiento que consta de dos etapas: en primer lugar se sintetiza un derivado de uretano-metacrilato a partir de hidroxietilmetacrilato (HEMA) y tolueno-2,4-diisocianato y, a continuación, se hace reaccionar este derivado con $\beta$-CD [163]. La copolimerización del monómero de $\beta$-CD con HEMA conduce a hidrogeles que presentan una elevada capacidad de carga de ácido salicílico, sulfatiazol, rifampicina y naranja de metilo. El efecto sobre la velocidad de cesión resultó ser dependiente de la hidrofilia del fármaco, ralentizándose ligeramente la liberación de naranja de metileno y ácido salicílico, y acelerándose la del fármaco hidrofóbico sulfatiazol (Figura 1.18). Las diferencias en la solubilidad y en la afinidad de los fármacos por las CDs explican los efectos contrapuestos que se derivan de la incorporación del monómero de $\beta$-CD al hidrogel.

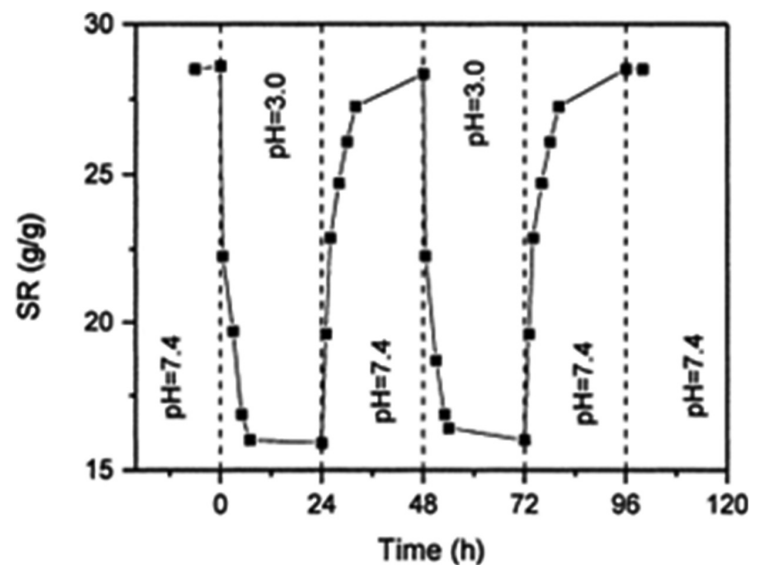

Figura 1.17. Respuesta al $\mathrm{pH}$ de hidrogeles PNIPA-co-MAH- $\beta$-CD. Reproducido de referencia [159] con permiso de Elsevier. 


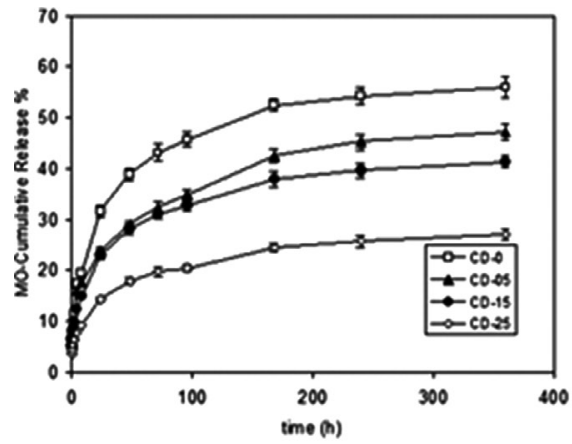

(a)

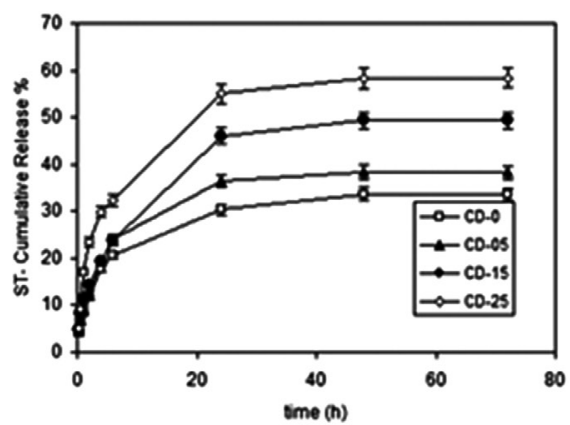

(c)

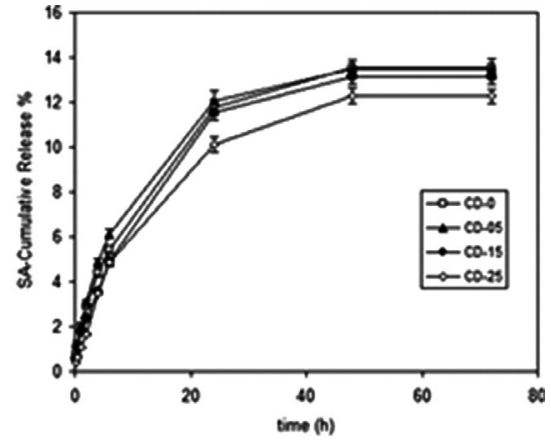

(b)

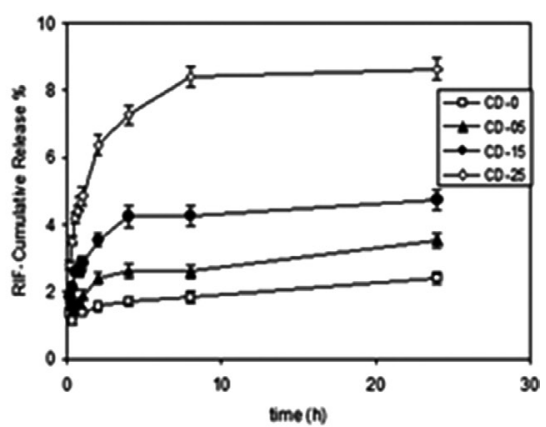

(d)

Figura 1.18. Perfiles de liberación de naranja metilo (a), ácido salicílico (b), sulfatiazol (c) y rifampicina (d) a partir de hidrogeles de $\beta$-CD-UM. Reproducido de referencia [163] con permiso de John Wiley and Sons.

Los monómeros metacrílicos de CDs despiertan un gran interés por las posibilidades que ofrecen en una gran variedad de campos. Se pueden obtener monómeros de CDs con 6 dobles enlaces en una sola etapa, haciendo reaccionar $\beta$-CD con anhídrido metacrílico [164], y monómeros de CD con grupos metacrílicos en las posiciones 2 y 3 en dos etapas que comprenden la acetilación de los grupos hidroxilo primarios y la posterior esterificación de los grupos hidroxilo secundarios con anhídrido metacrílico [165]. La esterificación completa de los grupos hidroxilo primarios (7) y secundarios (14) permite preparar el monómero (2,3-di-O-metacrilato-6metacrilato)- $\beta-C D$ [166] (Figura 1.15i). Al contar con numerosos grupos reactivos, el monómero metacrilato- $\beta C D$ actúa como agente reticulante, incrementando la rigidez de entramados de HEMA, lo que limita su capacidad para incorporar agua [167]. Estos hechos determinan que sólo 
cuando se incorpora el monómero metacrilato- $\beta C D$ en proporciones bajas se pueda manifestar una mejora en la carga de fármacos como hidrocortisona o acetazolamida [167].

Los monómeros de metacrilato también se pueden utilizar como rellenos para obturación/reconstrucción dental. Algunos fotoiniciadores, como la camforquinona y el etil-4-dimetilaminobenzoato, forman complejos de inclusión con los monómeros, alterando sustancialmente la fuerza de adhesión y el grado de conversión durante la consolidación del relleno [168,169]. Ajustando la relación grupos metacrilato polimerizables/grupos hidroxilo se pueden obtener monómeros de CD adhesivos que promueven el anclaje del empaste a la dentina. Las resinas preparadas con un 33\% de $\beta$-CD metacrilada, 30\% HEMA y 37\% acetona ofrecen una resistencia a la fuerza de cizalla similar a la de empastes comercializados [170].

\subsection{Funcionalización de entramados preformados con CDs}

La funcionalización de materiales preformados es un área en la que se está desarrollando una investigación cada vez más intensa. Los biomateriales deben combinar propiedades estructurales y superficiales aptas para la aplicación a la que se destinan. Ciertas propiedades estructurales, como la resistencia física y la estabilidad química, determinan la durabilidad del material, al tiempo que las características superficiales condicionan la naturaleza y la intensidad de las interacciones cuando el material entra en contacto con otros materiales o con tejidos vivos. La funcionalización superficial con CDs abre un abanico de posibilidades a la hora de modular la afinidad de la superficie por determinadas moléculas. Por ejemplo, se pueden anclar CDs a materiales textiles para que retengan colores, esencias, repelentes de insectos o agentes antimicrobianos [171-174]. La modificación superficial de materiales que se utilizan en la fabricación de dispositivos médicos con CDs reduce la adsorción de proteínas, los hace más hemocompatibles y abre la posibilidad de incorporar fármacos [175-177]. 
El anclaje de CDs a hidrogeles preformados permite dotarlos de capacidad para formar complejos con moléculas activas sin alterar otras propiedades. Este aspecto es particularmente relevante porque, como se mencionó anteriormente, los monómeros de CD suelen presentar varios dobles enlaces reactivos y actúan como agentes reticulantes modifcando las propiedades viscoelásticas, mecánicas y de hinchamiento del hidrogel. Se han diseñado hidrogeles capaces de experimentar de forma autónoma transiciones de volumen (ciclos de hinchamiento/ contracción) en los que la $\beta$-CD actúa como sensor de una sustancia y la NIPA se comporta como actuador [178]. Para ello, se copolimerizó NIPA (20 g) con p-nitrofenolacrilato $(3.4 \mathrm{~g})$ en $\mathrm{N}, \mathrm{N}^{\prime}$-dimetilformamida y, a continuación, se incorporaron CDs aminadas a los grupos p-nitrofenilacrilato (Figura 1.19).

La complejación de la CD con ácido 8-anilino-1-naftaleno-sulfónico altera el equilibrio hidrofílico/hidrofóbico, reduce la temperatura de transición y hace posible que se contraiga el entramado polimérico (Figura 1.19a). La contracción desestabiliza el complejo provocando la decomplejación, con lo que se restablece la temperatura de transición y el hidrogel se hincha. La coordinación de estos dos efectos (complejación/contracción) a una temperatura intermedia entre la de transición del hidrogel cuando las CDs están formando complejos, y la de transición cuando las CDs se encuentran libres, conduce a cambios autónomos de volumen (Figura 1.19b). Estos hidrogeles podrían ser útiles como sensores.

También se han incorporado CDs a hidrogeles preformados de HEMA copolimerizados con glicildilmetacrilato (GMA). Este último monómero presenta un grupo epóxido capaz de formar enlaces éter con los grupos hidroxilo de las CDs [179, 180]. De este modo se obtiene un entramado en el que las CDs no forman parte de su estructura primaria, sino que se encuentran unidas por 2-3 enlaces éter (Figura 1.20). 
a)

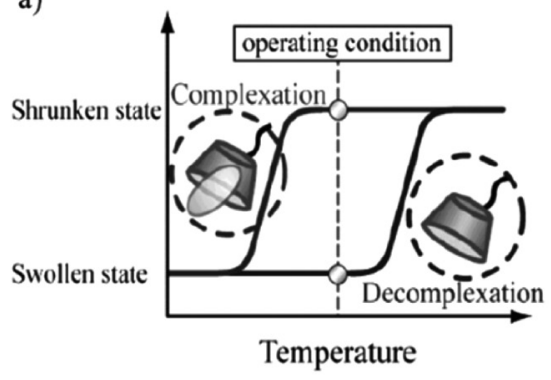

b)

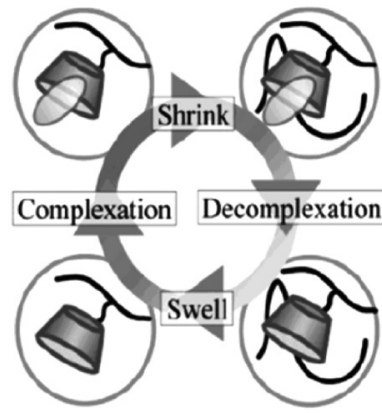

Figura 1.19. (a) Cambios de volumen inducidos por modificaciones en la temperatura de un hidrogel de poli(NIPA-Co-CD) en presencia de 8-anilino-1naftaleno-sulfónico, y (b) esquema del fenómeno oscilatorio autónomo: contracción del polímero, decomplejación del 8-anilino-1-naftaleno-sulfonico, hinchamiento del polímero, y formación del complejo con el 8-anilino-1-naftaleno-sulfonico. Reproducido de referencia [178] con permiso de la American Chemical Society.

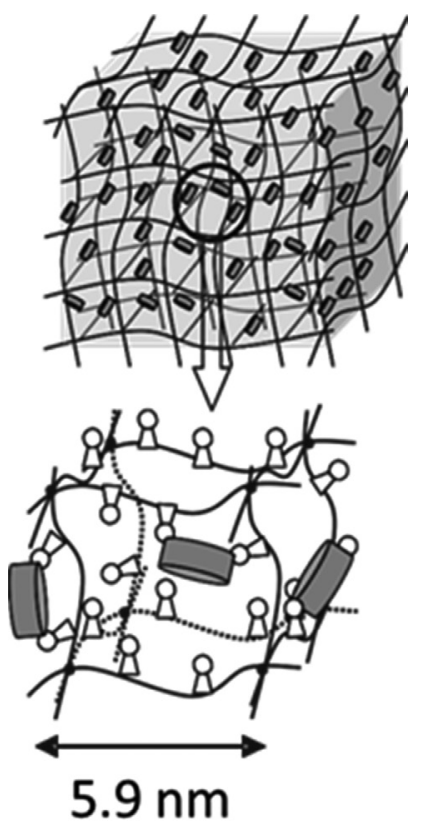

Figura 1.20. Esquema de un hidrogel de pHEMA-co-GMA con $\beta C D$ s colgantes. Reproducido de referencia [180] con permiso de Elsevier.

Este procedimiento permite desarrollar lentes de contacto blandas medicadas que desempeñan su función primaria de correctores de la 
visión y al mismo tiempo actúan como depots de fármaco sobre la superficie ocular. La incorporación de $\beta$-CDs colgantes permitió incrementar 15 veces la afinidad por diclofenaco y los hidrogeles sostuvieron la cesión en fluido lacrimal durante 2 semanas [180]. Por otra parte parte, hidrogeles similares con $\gamma$-CDs ancladas incorporaron cantidades de nitrato de miconazol suficientes para inhibir el crecimiento de Candida albicans [181].

\subsection{Conclusiones}

Las CDs ofrecen grandes posibilidades para modular la velocidad de cesión de fármacos a partir de hidrogeles físicos o covalentemente reticulados. Su capacidad para formar complejos de inclusión permite formular fármacos hidrofóbicos en sistemas hidrofílicos. Cuando las CDs se encuentran libres en el sistema, la complejación tiene como resultado, además, una modificación del tamaño hidrodinámico de las especies que difunden y de las interacciones del fármaco con las cadenas poliméricas. El balance de estos efectos determina la velocidad de cesión. Por otra parte, la reticulación directa de CDs, su incorporación a entramados acrílicos formando parte del esqueleto polimérico o su anclaje a entramados preformados conduce a hidrogeles con dominios de CDs con una concentración local que no se modifica significativamente al entrar en contacto con los fluidos biológicos. Esto permite maximizar la capacidad de las CDs para formar complejos de inclusión y, por lo tanto, dota a los entramados de capacidad de control de la cesión por afinidad. La versatilidad de los procedimientos de preparación de hidrogeles, tanto a escala macro como nanoscópica, abre unas perspectivas muy favorables para incorporar CDs en estructuras muy diversas y con capacidad de carga y de control de la cesión adecuadas para hacer frente a necesidades especificas tanto en el desarrollo de nuevos sistemas de liberación de fármacos como de sistemas de combinación fármacoproducto sanitario. 


\subsection{Bibliografía}

[1] C.A. Lipinski, J. Pharmacol. Toxicol. Meth. 2000, 44, 235-249.

[2] T. Takagi, C. Ramachandran, M. Bermejo, S. Yamashita, L.X. Yu, G.L. Amidon, Mol. Pharm. 2006, 3, 631-643.

[3] K.Y. Lee, S.H. Yuk, Prog. Polymer Sci. 2007, 32, 669-697.

[4] C. Alvarez-Lorenzo, A. Concheiro, Mini-Rev. Med. Chem. 2008, 8, 1065-1074.

[5] M.E. Davis, M.E. Brewster, Nature Rev. 2004, 3, 1023-1035.

[6] K. Uekama, F. Hirayama, T. Irie, Chem. Rev. 1998, 98, 2045-2076.

[7] C. Rodriguez-Tenreiro, C. Alvarez-Lorenzo, A. Rodriguez-Perez, A. Concheiro, J.J. TorresLabandeira, Pharm. Res. 2006, 23, 121-130.

[8] T. Loftsson, D. Duchene, Int. J. Pharm. 2007, 329, 1-11.

[9] A.A. Hincal, H. Eroglu, E. Bilensoy, in: Cyclodextrins in Pharmaceutics, Cosmetics and Biomedicine. Current and future industrial applications, E. Bilensoy (Ed.), John Wiley and Sons, Hoboken, NJ, 2011, 123-130.

[10] J. Szejtli, Chem. Rev. 1998, 98, 1743-1753.

[11] J. Blanchard, S. Proniuk, Pharm. Res. 1999, 16, 1796-1798.

[12] M.E. Brewster, T. Loftsson, Adv. Drug Deliv. Rev. 2007, 59, 645-666.

[13] L. Liu, Q.X. Guo, J. Incl. Phenom. Macro. 2002, 42, 1-14.

[14] D.O. Thompson, Crit. Rev. Therap. Drug Carrier Syst. 1997, 14, 1-104.

[15] T. Loftsson, M. Másson, J.F. Sigurjónsdótirr, STP Pharma. Sci. 1999, 9, 237-242.

[16] T Loftsson, M. Másson, Int. J. Pharm. 2001, 225, 15-30.

[17] G.L. Perlovich, M. Skar, A. Bauer-Brandl, Eur. J. Pharm. Sci. 2003, 20, 197-200.

[18] T. Loftsson, D. Hreinsdóttir, M. Másson, Int. J. Pharm. 2005, 302, 18-28.

[19] J. Szejtli, L. Szente, Eur. J. Pharm. Biopharm. 2005, 61, 115-125.

[20] E. Irie, K. Uekama, J. Pharm. Sci. 1997, 86, 147-162.

[21] H.W. Frijlink, A.C. Eissens, A.J.M. Schoonen, C.F. Lerk, Int. J. Pharm. 1990, 64, 195-205.

[22] D. Amdidouche, P. Montassier, M.C. Poelman, D. Duchene. Int. J. Pharm. 1994, 111, 111-116.

[23] K. Uekama, Chem. Pharm. Bull. 2004, 52, 900-915.

[24] V.J. Stella, R.A. Rajewski, Pharm. Res. 1997, 14, 556-567.

[25] J. Szejtli, Pure Appl. Chem. 2004, 76, 1825-1845.

[26] T. Loftsson, P. Jarho, M. Másson, T. Jarvinen, Expert Opin. Drug Deliv. 2005, 2, 335-351.

[27] R. Challa, A. Ahuja, J. Ali, R.K. Khar, AAPS PharmSciTech 2005, 6, Article 43, E329-E357.

[28] T. Loftsson, M.E. Brewster, M. Másson, Am. J. Drug Deliv. 2004, 2, 261-275.

[29] G.L. Amidon, H. Lennernäs, V.P. Shah, J.R. Crison, Pharm. Res. 1995, 12, 413-420.

[30] E. Gupta, D.M. Barends, E. Yamashita, Eur. J. Pharm Sci. 2006, 29, 315-324.

[31] H.M. Cabral Marques, Rev. Port. Farm. 1994, 44, 77-84.

[32] K. Uekama, F. Hirayama, T. Irie, Drug Target. Del. 1994, 3, 411-456.

[33] V.J. Stella, V.M. Rao, E.A. Zannou, V.Zia, Adv. Drug Del. Rev. 1999, 36, 3-16. 
[34] T. Jarvinen, K. Jarvinen, N. Schwarting, V.J. Stella, J. Pharm. Sci. 1995, 84, 295-299.

[35] A. Géze, S. Aous, I. Baussanne, J.L. Putaux, J.Defaye, D. Wouessidjewe, Int. J. Pharm. 2002, 242, 301-305.

[36] A. Magnusdottir, M. Másson, T. Loftsson, J. Incl. Phenom. Macro. 2002, 44, 213-218.

[37] T. Loftsson, M. Másson, M.E. Brewster, J. Pharm. Sci. 2004, 93, 1091-1099.

[38] K. Uekama, T. Horikawa, Y. Horiuchi, F. Hirayama, J. Control. Release 1993, 25, 99-106.

[39] F. Hirayama, K. Uekama, Adv. Drug Del. Rev. 1999, 36, 125-141.

[40] T. Horikawa, F. Hirayama, K. Uekama, J. Pharm. Pharmacol. 1995, 47, 124-127.

[41] Y. Ikeda, K. Kimura, F. Hirayama, H. Arima, K. Uekama, J. Control. Release 2000, 66, 271-280.

[42] N.A. Peppas, P. Bures, W. Leobandung, H. Ichikawa, Eur. J. Pharm. Biopharm. 2000, 50, 27-46.

[43] C. Alvarez-Lorenzo, J.L. Gomez-Amoza, R. Martinez-Pacheco, C. Souto, A. Concheiro, Int. J. Pharm. 1999, 180, 91-105.

[44]R. Barreiro-Iglesias, C. Alvarez-Lorenzo, A. Concheiro, J. Control. Release 2001, 77, 5975.

[45] T.R. Hoare, D.S. Kohane, Polymer 2008, 49, 1993-2007.

[46] D.C. Bibby, N.M. Davies, I.G. Tucker, Int. J. Pharm. 2000, 197, 1-11.

[47] R.J. Babu, P. Dayal, M. Singh, Drug Del. 2008, 15, 381-388.

[48] F. Quaglia, G. Varricchio, A. Miro, M.I. La Rotonda, D. Mensitieri, G. Mensitieri, Acta Technol. Legis. Medicam. 2001, 12, 215-224.

[49] V.M. Rao, J.L. Haslam, V.J. Stella, J. Pharm. Sci. 2001, 90, 807-816.

[50] U. Werner, C. Damgé, P. Maincent, R. Bodmeier, J. Drug Del. Sci. Tech. 2004, 14, 275284.

[51] I. Orienti, V. Zecchi, G. Ceschel, A. Fini, Eur. J. Drug Metab. Ph. 1991, 466-472.

[52] F. Quaqlia, G. Varrichio, A. Miro, M.I. La Rotonda, D. Larobina, G. Mensitieri, J. Control. Release 2001, 71, 329-337.

[53] L.S. Koester, C.R. Xavier, P. Mayorga, V.L. Bassani, Eur. J. Pharm. Biopharm. 2003, 55, 85-91.

[54] A. Doliwa, S. Santoyo, P. Ygartua, Drug Dev. Ind. Pharm. 2001, 27, 751-758.

[55] H.S. Woldum, F. Madsen, K.L. Larsen, Drug Del. 2008, 15, 69-80.

[56] H. Blanco-Fuente, B. Esteban-Fernandez, J. Blanco-Mendez, F.J. Otero-Espinar, Chem. Pharm. Bull. 2002, 50, 40-46.

[57] B. Pose-Vilarnovo, L. Santana-Penin, M. Echezarreta-Lopez, M.B. Perez-Marcos, J.L. Vila-Jato, J.J. Torres-Labandeira, STP Pharma Sci. 1999, 9, 231-236.

[58] B. Pose-Vilarnovo, M. Echezarreta-Lopez, P. Schroth-Pardo, E. Estrada, J.J. TorresLabandeira, Eur. J. Pharm. Sci. 2001, 13, 325-331.

[59] B. Pose-Vilarnovo, C. Rodriguez-Tenreiro, J.F. Rosa dos Santos, J. Vazquez-Doval, A. Concheiro, C. Alvarez-Lorenzo, J.J. Torres-Labandeira, J. Control. Release 2004, 94, 351363.

[60] A.E. Tonelli, Polymer 2008, 49, 1725-1736.

[61] P. Zheng, X. Hu, X. Zhao, L. Li, K.C. Tam, L.H. Gan, Macromol. Rapid Commun. 2004, $25,678-682$. 
[62] C. Alvarez-Lorenzo, S. Deshmukh, L. Bromberg, T.A. Hatton, I. Sandez-Macho, A. Concheiro, Langmuir 2007, 23, 11475-11481.

[63] S. Loethen, J.M. Kim, D.H. Thompson, Polymer Rev. 2007, 47, 383-418.

[64] M. Okada, M. Kamachi, A. Harada, J. Phys. Chem. B 1999, 103, 2607-2613.

[65] M. Kidowaki, C. Zhao, T. Kataoka, K. Ito, Chem. Commun. 2006, 39, 4102-4103.

[66] A. Kikuzawa, T. Kida, M. Akashi, Macromolecules 2008, 41, 3393-3395.

[67] K. Karaky, C. Brochon, G. Schlatter, G. Hadziioannou, Soft Mater. 2008, 4, 1165-1168.

[68] K. Tamura, K. Hatanaka, N. Yoshie, Polymer Int. 2007, 56, 1115-1121.

[69] F. Huang, H.W. Gibson, Prog. Polym. Sci. 2005, 30, 982-1018.

[70] A. Yamashita, D. Kanda, R. Katoono, N. Yui, T. Ooya, A. Maruyama, H. Akita, K. Kogure, H. Harashima, J. Control. Release 2008, 131, 137-144.

[71] T. Ooya, N. Yui, Crit. Rev. Therap. Drug Carrier Syst. 1999, 16, 289-330.

[72] A.I. Rodriguez-Perez, C. Rodriguez-Tenreiro, C. Alvarez-Lorenzo, A. Concheiro, J.J. Torres-Labandeira, J. Nanosci. Nanotechnol. 2006, 6, 3179-3186.

[73] A.I. Rodriguez-Perez, C. Rodriguez-Tenreiro, C. Alvarez-Lorenzo, A. Concheiro, J.J. Torres-Labandeira, J. Incl. Phenom. Macro. 2007, 57, 497-501.

[74] G. Gonzalez-Gaitano, W. Brown, G. Tardajos, J. Phys. Chem. B 1997, 101, 710-719.

[75] J. Li, X. J. Loh, Adv. Drug Del. Rev. 2008, 60, 1000-1017.

[76] L. Nogueiras-Nieto, C. Alvarez-Lorenzo, I. Sandez-Macho, A. Concheiro, F.J. Otero-Espinar, J. Phys. Chem. B 2009, 113, 2773-2782.

[77] G. Wenz, M. Weickenmeier, J. Huff, ACS Symposium Series 2000, 765, 271-283.

[78] R. Auzely-Velty, M. Rinaudo, Macromolecules 2002, 35, 7955-7962.

[79] A. Hashidzume, F. Ito, I. Tomatsu, A. Harada, Macromol. Rapid Commun. 2005, 26, 1151-1154.

[80] V. Wintgens, M. Charles, F. Allouache, C. Amiel, Macromol. Chem. Phys. 2005, 206, 1853-1861.

[81] S. Daoud-Mahammed, J.L. Grossiord, T. Bergua, C. Amiel, P. Couvreur, R. Gref, J. Biomed. Mater. Res. 2007, 86A, 736-748.

[82] V. Wintges, S. Daoud-Mahammed, R. Gref, L. Bouteiller, C. Amiel, Biomacromolecules 2008, 9, 1434-1442.

[83] R. Gref, C. Amiel, K. Molinard, S. Daoud-Mahammed, B. Sébille, B. Gillet, J.C. Beloeil, C. Ringard, V. Rosilio, J. Poupaert, P. Couvreur, J. Control. Release 2006, 111, 316-324.

[84] V. Wintgens, T.T. Nielsen, K.L. Larsen, C. Amiel, Macromol. Biosci. 2011, 11, 1254-1263.

[85] J. Szeman, E. Fenyvesi, J. Szejtli, H. Ueda, Y. Machida, T. Nagai, J. Incl. Phenom. 1987, 5, 427-31.

[86] G. Crini, S. Bertini, G. Torri, A. Naggi, D. Sforzini, C. Vecchi, L. Janus, Y. Lekchiri, M. Morcellet, J. Appl. Polym. Sci. 1998, 68, 1973-1978.

[87] A.M. Layre, N.M. Gosselet, E. Renard, B. Sebille, C. Amiel, J. Incl. Phenom. Macro. 2002, $43,311-317$.

[88] J. Li, H. Xiao, J. Li, Y.P. Zhong, Int. J. Pharm. 2004, 278, 329-342.

[89] Y. Liu, Y.W. Yang, E.C. Yang, X.D. Guan, J. Org. Chem. 2004, 69, 6590-6602.

[90] C. Gazpio, M. Sanchez, J.R. Isasi, I. Velaz, C. Martin, C. Martinez-Oharriz, A. Zornoza, Carbohyd. Polym. 2008, 71, 140-146. 
[91] L. Qian, Y. Guan, H. Xiao, Int. J. Pharm. 2008, 357, 244-251.

[92] G. Crini, M. Morcellet, J. Sep. Sci. 2002, 25, 789-813.

[93] W. L. Xu, J.D. Liu, Y.P. Sun, Chinese Chem. Lett. 2003, 14, 767-770.

[94] A. Yudiarto, S. Kashiwabara, Y. Tashiro, T. Kokugan, Sep. Pur. Tech. 2001, 24, 243-253.

[95] G. Crini, C. Cosentino, S. Bertini, A. Naggi, G. Torri, C. Vecchi, L. Janu, M. Morcellet, Carbohydr. Res. 1998, 308, 37-45.

[96] I. Velaz, J.R. Isasi, M. Sánchez, M. Uzqueda, G. Ponchel, J. Incl. Phenom. Macro. 2007, $57,65-68$.

[97] R. Orprecio, C.H. Evans, J. Appl. Polym. Sci. 2003, 90, 2103-2110.

[98] G. Crini, Prog. Polym. Sci. 2005, 30, 38-70.

[99] G. Crini, Dyes Pigm. 2008, 77, 415-426.

[100] C.H. Su, C.P. Yang, J. Sci. Food Agric. 1991, 54, 635-643.

[101] E. Schneiderman, A.M. Stalcup, J. Chromatogr. B 2000, 745, 83-102.

[102] G.K.E. Scriba, J. Sep. Sci. 2008, 31, 1991-2011.

[103] N. Morin-Crini, G. Crini, Prog. Polym. Sci. 2013, 38, 344-368.

[104] N. Wiedenhof, J.N.J.J. Lammers, C.L. Van Panthaleon van Eck, Starch-Stärke 1969, 21, 119-123.

[105] J.L. Hoffman, J. Macro. Sci. Chem. 1973, A7, 1147-1157.

[106] A. Harada, M. Furue, S. Nozakur, J. Polym. Sci- Polym. Chem. Ed. 1978, 16, 189-196.

[107] N. Thuaud, B. Sebille, E. Renard, J. Biochem. Bioph. Meth. 2002, 54, 327-337.

[108] H.D. Wang, L.Y. Chu, H. Song, J.P. Yang, R. Xie, M. Yang, J. Membr. Sci. 2007, 297, 262-270.

[109] J. Szejtli, E. Fenyvesi, B. Zsadon, Starch/Stärke. 1978, 30, 127-131.

[110] E. Fenyvesi, A. Ujhazy, J. Szejtli, S.Pütter, T.G. Gan, J. Inclus. Phenom. Mol. 1996, 25, 443-447.

[111] T. Nozaki, Y. Maeda, H. Kitanao, J. Polym. Sci. Pol. Chem. 1997, 35, 1535-1541.

[112] J.T. Zhang, S.W. Huang, F.Z. Gao, R.X. Zhuo, Colloid Polym. Sci. 2005, 283, 461-464.

[113] J.T. Zhang, Y.N. Xue, F.Z. Gao, S.W. Huang, R.X. Zhuo, J. Appl. Polym. Sci. 2008, 108, 3031-3037.

[114] Y.Y. Liu, X.D. Fan, T. Kang, L. Sun, Macromol. Rapid Comm. 2004, 25, 1912-1916.

[115] Z.W. Gao, X.P. Zhao, Polymer 2003, 44, 4519-4526.

[116] Z.W. Gao, X.P. Zhao, J. Appl. Polym. Sci. 2004, 93, 1681-1686.

[117] H.A. Tsai, M.J. Syu, Biomaterials. 2005, 26, 2759-2766.

[118] W.E. Baille, W.Q. Huang, M. Nichifor, X.X. Zhu, J. Macromol. Sci. A 2000, 37, 677-690.

[119] G. Mocanu, D. Vizitiu, A. Carpov, J. Bioact. Compatible Polym. 2001, 16, 315-342.

[120] H. Yamasaki, Y. Makihata, K. Fukunaga, J. Chem. Technol. Biotechnol. 2008, 83, 991997.

[121] E. Y. Ozmen, M. Sezgin, A. Yilmaz, M. Yilmaz. Bioresour. Technol. 2008, 99, 526-531.

[122] S. Salmaso, A. Semenzato, S. Bersani, P. Matricardi, F. Rossi, P. Caliceti, Int. J. Pharm. 2007, 345, 42-50.

[123] L. Chen, X. Zhu, D. Yan, X. He, Polym. Prepr. 2003, 44, 669-670. 
[124] M. Ma, D.Q. Li, Chem. Mater. 1999, 11, 872-874.

[125] C. Alvarez-Lorenzo, A. Concheiro, J. Chromatogr. B 2004, 804, 231-245.

[126] H. Asanuma, T. Hishiya, M. Komiyama. J. Incl. Phenom. Macro. 2004, 50, 51-55.

[127] T. Hishiya, M. Shibata, M. Kakazu, H. Asanuma, M. Komiyama, Macromolecules 1999, $32,2265-2269$.

[128] T. Hishiya, H. Asanuma, M. Komiyama, J. Am. Chem. Soc. 2002, 124, 570-575.

[129] I.X. Garcia-Zubiri, G. Gonzalez-Gaitano, J.R. Isasi, J. Colloid Interf. Sci. 2007, 307, 64-70.

[130] A. Romo, F.J. Penas, J.R. Isasi, I.X. Garcia-Zubiri, G. Gonzalez-Gaitano, React. Funct. Polym. 2008, 68, 406-413.

[131] N. Pariot, F. Edwards-Levy, M.C. Andry, M.C. Levy, Int. J. Pharm. 2000, 211, 19-27.

[132] N. Pariot, F. Edwards-Levy, M.C. Andry, M. C. Levy, Int. J. Pharm. 2002, 232, 175-181.

[133] F. Trotta, R. Cavalli, Compos. Interface. 2009, 16, 39-48.

[134] K.A. Ansari, P.R. Vavia, F. Trotta, R. Cavalli, AAPS PharmSciTech. 2011, 12, 279-286.

[135] B. Martel, D. Ruffin, M. Weltrowski, Y. Lekchiri, M. Morcellet, J. Appl. Polym. Sci. 2005, 97, 433-442.

[136] C. Alvarez-Lorenzo, C. Rodriguez-Tenreiro, J.J. Torres-Labandeira, A. Concheiro, PCT Int. Appl. 2006, WO 2006089993.

[137] N. Yui, T. Okano, J. Control. Release 1992, 22, 105-116.

[138] L.L.H. Huang, P.C. Lee, L.W. Chen, K.H. Hsieh, J. Biomed. Mater. Res. 1998, 39, 630636.

[139] C. Rodriguez-Tenreiro, C. Alvarez-Lorenzo, A. Rodriguez-Perez, A. Concheiro, J.J. Torres-Labandeira, Eur. J. Pharm. Biopharm. 2007, 66, 55-62.

[140] E. Lopez-Montero, C. Alvarez-Lorenzo, J.F. Rosa dos Santos, J.J. Torres-Labandeira, A. Concheiro, Open Drug Deliv. J. 2009, 3, 1-9.

[141] B. Blanco-Fernandez, M. Lopez-Viota, A. Concheiro, C. Alvarez-Lorenzo, Carbohydr. Polym. 2011, 85, 765-774.

[142] M.D. Moya-Ortega, C. Alvarez-Lorenzo, H.H. Sigurdsson, A. Concheiro, T. Loftsson, Carbohydr. Polym. 2011, 87, 2344-2351

[143] C. Rodriguez-Tenreiro, L. Diez-Bueno, A. Concheiro, J.J. Torres-Labandeira, C. AlvarezLorenzo, J. Control. Release 2007, 123, 56-66.

[144] A. Harada, M. Furue, S. Nozakura, Macromolecules 1976, 9, 701-704.

[145] A. Harada, M.Furue, S. Nozakura, Macromolecules 1976, 9, 705-710.

[146] J.T. Zhang, S.W. Huang, R.X. Zhuo, Macromol. Chem. Phys. 2004, 205, 107-113.

[147] H. Asanuma, T. Akiyama, K. Kajiya, T. Hishiya, M. Komiyama, Anal. Chim. Acta 2001, $435,25-33$.

[148] S.A. Piletsky, H.S. Andersson, I.A. Nicholls, Macromolecules 1999, 32, 633-636.

[149] S.A. Piletsky, H.S. Andersson, I.A. Nicholls, Polymer J. 2005, 37, 793-796.

[150] T. Seo, T. Kajihara, T. Iijima, Macromol. Chem. 1987, 188, 2071-2082.

[151] G. Crini, G. Torri, M. Guerrini, B. Martel, Y. Lekchiri, M. Morcellet, Eur. Polym. J. 1997, 33, 1143-1151.

[152] H.L. Ramirez, R. Cao, A. Fragoso, J.J. Torres-Labandeira, A. Dominguez, E.H. Schacht, M. Baños, R. Villalonga, Macromol. Biosci. 2006, 6, 555-561. 
[153] Y.Y. Liu, X.D. Fan, L. Gao, Macromol. Biosci. 2003, 3, 715-719.

[154] M.H. Lee, K.J. Yoon, S.H. Ko, J. Appl. Polym. Sci. 2001, 80, 438-446.

[155] U. Siemoneit, C. Schmitt, C. Alvarez-Lorenzo, A. Luzardo, F. Otero-Espinar, A. Concheiro, J. Blanco-Mendez, Int. J. Pharm. 2006, 312, 66-74.

[156] T. Osawa, K. Shirasaka, T.Matsui, S. Yoshihara, T. Akiyama, T. Hishiya, H. Asanuma, M. Komiyama, Macromolecules 2006, 39, 2460-2466.

[157] S.H. Song, K. Shirasaka, Y. Hirokawa, H. Asanuma, T. Wada, J. Sumaoka, M. Komiyama, Supramol. Chem. 2010, 22, 149-155.

[158] S. Song, K. Shirasaka, M. Katayama, S. Nagaoka, S. Yoshihara, T. Osawa, J. Sumaoka, H. Asanuma, M. Komiyama, Macromolecules 2007, 40, 3530-3532.

[159] Y.Y. Liu, X.D. Fan, Polymer 2002, 43, 4997-5003.

[160] Y.Y. Liu, X.D. Fan, H. Hu, Z.H. Tang, Macromol. Biosci. 2004, 4, 729-736.

[161] D. Ma, L.M. Zhang, C. Yang, L. Yan, J. Polym. Res. 2008, 15, 301-307.

[162] D. Lu, L. Yang, T. Zhou, Z. Lei, Eur. Polym. J. 2008, 44, 2140-2145.

[163] S. Demir, M.V. Kahraman, N. Bora, N.K. Apohan, A. Ogan, J. Appl. Polym. Sci. 2008, 109, 1360-1368.

[164] S.A. Zawko, C.E. Schmidt, Polym. Mater. Sci. Eng. 2006, 95, 1022-1023.

[165] R. Saito, Y. Okuno, H. Kobayashi, J. Polym. Sci. A 2001, 39, 3539-3576.

[166] R. Saito, K. Yamaguchi, Macromolecules 2003, 36, 9005-9013.

[167] J.F. Rosa dos Santos, R. Couceiro, A. Concheiro, J.J. Torres-Labandeira, C. AlvarezLorenzo, Acta Biomater. 2008, 4, 745-755.

[168] L.A. Hussain, S.H. Dickens, R.L. Bowen, Dent. Mater. 2004, 20, 513-521.

[169] L.A. Hussain, S.H. Dickens, R.L. Bowen, Dent. Mater. 2005, 21, 210-216.

[170] L.A. Hussain, S.H. Dickens, R.L. Bowen, Biomaterials 2005, 26, 3973-3979.

[171] E. Hiriart-Ramírez, A. Contreras-García, M.J. Garcia-Fernandez, A. Concheiro, C. AlvarezLorenzo, E. Bucio, Cellulose, 2012, 19, 2165-2177.

[172] R. Romi, P. Lo Nostro, E. Bocci, F. Ridi, P. Baglioni, Biotechnol. Prog. 2005, 21, 1724-1730.

[173] A. Hebeish, M.M.G. Fouda, I.A. Hamdy, S.M. El-Sawy, F.A. Abdel-Mohdy, Carbohyd. Polym. 2008, 74, 268-273.

[174] C.X. Wang, S.L. Chen, Appl. Surf. Sci. 2006, 252, 6348-6352.

[175] X. Zhao, J.M. Courtney, J. Biomed. Mater. Res. 2007, 80A, 539-553.

[176] C.A.B. Nava-Ortiz, C. Alvarez-Lorenzo, E. Bucio, A. Concheiro, G. Burillo, Int. J. Pharm. 2009, 382, 183-191

[177] C.A.B. Nava-Ortiz, G. Burillo, A. Concheiro, E. Bucio, N. Matthijs, H. Nelis, T. Coenye, C. Alvarez-Lorenzo, Acta Biomater. 2010, 6, 1398-1404.

[178] H. Ohashi, Y. Hiraoka, T. Yamaguchi, Macromolecules 2006, 39, 2614-2620.

[179] T. Shan, J. Chen, L. Yang, S. Jie, Q. Qian, J. Radioanal. Nucl. Chem. 2009, 279, 75-82.

[180] J.F. Rosa dos Santos, C. Alvarez-Lorenzo, M. Silva, L. Balsa, J. Couceiro, J.J. TorresLabandeira, A. Concheiro, Biomaterials 2009, 30, 1348-1355.

[181] J.F. Rosa dos Santos, J.J. Torres-Labandeira, N. Matthijs, T. Coenye, A. Concheiro, C. Alvarez-Lorenzo, Acta Biomater. 2010, 6, 3919-3916. 